\title{
Charge and Thermoelectric Transport in Polymer-Sorted Semiconducting Single-Walled Carbon Nanotube Networks
}

\author{
Martin Statz, ${ }^{\dagger}$ Severin Schneider, ${ }^{\ddagger}$ Felix J. Berger, ${ }^{\ddagger}$ Lianglun Lai, ${ }^{\dagger}, \boldsymbol{\Phi}$ William A. \\ Wood, ${ }^{\dagger}$ Mojtaba Abdi-Jalebi, ${ }^{\dagger} \S$ Simone Leingang, $\|$ Hans-Jörg Himmel, \\ Zaumseil, ${ }^{*}, \perp$ and Henning Sirringhaus ${ }^{*, \dagger}$ \\ $\dagger$ Cavendish Laboratory, University of Cambridge, Cambridge CB3 0HE, UK \\ $\ddagger$ Institute for Physical Chemistry, Universität Heidelberg, D-69120 Heidelberg, Germany \\ \Cambridge Graphene Centre, University of Cambridge, Cambridge CB3 OFA, UK \\ $\S$ Institute for Materials Discovery, University College London, London WC1E 7JE, UK \\ || Institute for Inorganic Chemistry, Universität Heidelberg, D-69120 Heidelberg, Germany \\ $\perp$ Centre for Advanced Materials, Universität Heidelberg, D-69120 Heidelberg, Germany \\ E-mail: zaumseil@uni-heidelberg.de; hs220@cam.ac.uk
}

\section{Abstract}

Understanding the charge transport mechanisms in chirality-selected single-walled carbon nanotube (SWCNT) networks and the influence of network parameters is essential for further advances of their optoelectronic and thermoelectric applications. Here, we report on charge density and temperature-dependent field-effect mobility and on-chip field-effectmodulated Seebeck coefficient measurements of polymer-sorted monochiral small diameter $(6,5)$ $(0.76 \mathrm{~nm})$ and mixed large diameter SWCNT $(1.17-1.55 \mathrm{~nm})$ networks (plasma torch nanotubes, RN) with different network densities and length distributions. All untreated networks display balanced ambipolar transport and electron-hole symmetric Seebeck coefficients. We show that charge and thermoelectric transport in SWCNT networks can be modelled by the Boltzmann transport formalism, incorporating transport in heterogeneous media and fluctuation-induced tunneling. Considering the diameter-dependent one-dimensional density of states (DoS) of the SWCNTs composing the network, we can simulate the charge density and temperature-dependent Seebeck coefficients. Our simulations suggest that scattering in these networks cannot be described as simple one-dimensional acoustic and optical phonon scattering as for single SWCNTs. Instead the relaxation time is inversely proportional to energy $\left(\tau \propto\left(E-E_{\mathrm{C}}\right)^{s}, s=-1, E_{\mathrm{C}}\right.$ being the energy of the first van Hove singularity), presumably pointing towards the more two-dimensional character of scattering events and the necessity to include scattering at SWCNT junctions. Finally, our observation of higher power factors in trap-free, 1,2,4,5-tetrakis(tetramethylguanidino)benzene (ttmgb)-treated $(6,5)$ networks than in the RN networks, emphasizes the importance of chirality selection to tune the width of the DoS. In order to benefit from both higher intrinsic mobilities and a large thermally accessible DoS, we propose trap-free, narrow DoS distribution, large diameter SWCNT networks for both electronic and thermoelectric applications. 


\section{Keywords}

single-walled carbon nanotubes networks, charge transport, thermoelectric transport, Seebeck coefficient, Boltzmann transport formalism

Semiconducting single-walled carbon nanotube (SWCNT) networks are excellent candidates for next generation electronic devices due to their stability, flexibility, solutionprocessability as well as high carrier mobilities and Seebeck coefficients. ${ }^{1-4}$ Recently, the ability to tune the diameter distribution in these networks using selective dispersion with conjugated polymers and other separation methods has led to significant advances in electronic circuits and other applications. ${ }^{5-8} \mathrm{As}$ these highly selective sorting methods have only emerged lately, a complete understanding of the charge transport mechanisms in these networks is urgently desired to further improve device performance. ${ }^{9,10}$ Most existing transport models for SWCNT networks were developed before the advent of ultra-pure semiconducting networks without any metallic nanotubes. Those studies have focused on explaining transport in terms of fluctuation-induced tunneling, variable-range hopping and percolation theory. ${ }^{11-14}$ They mainly consider energetic disorder and sample heterogeneity effects, without explicitly considering the diameter distribution composing the networks. ${ }^{12,13,15}$ Nevertheless, studies on chirality-selected SWCNT networks have shown that the consideration of the relative contribution of different chiralities to charge transport is essential. ${ }^{9,16-18}$ A random resistor model of SWCNT junctions, considering the density of states (DoS) of individual SWCNTs but ignoring their resistance, and hence neglecting any intra-nanotube contributions, has been able to explain many qualitative features of the charge density dependence of the field-effect mobilities of mixed chirality SWCNT networks. This approach is rooted in percolation theory with variable-range hopping as a transport process and correctly predicts how with increasing charge density the filling of the first subband of the SWCNTs leads to a pronounced charge density dependence of the mo- bility in SWCNT networks. ${ }^{18-20}$ Furthermore, it demonstrates how small bandgap, large diameter SWCNTs dominate transport, particularly in the low charge density regime, in mixed chirality networks which has also been shown spectroscopically. ${ }^{17,18,21}$ However, we recently established that contrary to the expectation from this random resistor model, the temperature dependence of the mobility of large diameter mixed SWCNT (1.17-1.55 nm) networks, with a larger energetic disorder due to the bandgap distribution, is smaller than in monochiral $(6,5)$ SWCNT $(0.76 \mathrm{~nm})$ networks. ${ }^{9}$ This raises the question of potential phonon scattering-limited intra-nanotube transport contributions leading to the well-established dependence of carrier mobility on diameter $\left(\propto d^{2}\right)$ and temperature $\left(\propto T^{-1}\right) .{ }^{22,23}$ Hence, these findings necessitate further investigations of the influence of network parameters on the presumed transport mechanisms.

To establish the transport mechanisms in SWCNT networks with certain diameter distributions and to elucidate the influence of network parameters on charge and thermoelectric transport, we study the charge density and temperature-dependent field-effect mobility and on-chip field-effect-modulated Seebeck coefficient of polymer-sorted small diameter $(6,5)$ SWCNT $(0.76 \mathrm{~nm})$ networks as well as large diameter mixed SWCNT (1.17-1.55 nm) networks with different network densities and length distributions. We also establish the impact of trap states on transport by studying $(6,5)$ SWCNT networks that were treated with 1,2,4,5-tetrakis(tetramethylguanidino)benzene (ttmgb), which leads to pure electron transport. ${ }^{24}$ As the Seebeck coefficient is the entropy transported by a charge carrier divided by its charge, it provides detailed insights into the transport energetics and mechanisms. ${ }^{25,26}$ While the electrical conductivity has been shown to be predominantly limited by the highly resistive tunneling junctions, the Seebeck coefficient is expected to be much more sensitive to the energetics within the SWCNTs in the network, where most of the applied temperature drops. ${ }^{12,27}$ By using field-induced charge density-modulated measurements of the 
Seebeck coefficient we can eliminate the influence of the Coulombic interaction with counterions that would be present if carriers were introduced by molecular doping. ${ }^{28}$ These measurements allow us to establish a direct relationship between the DoS of the SWCNT networks and their Seebeck coefficients. Considering the diameter-dependent one-dimensional DoS of the network-composing SWCNTs from tight-binding nearest-neighbour calculations including trigonal warping effects, ${ }^{29,30}$ scaled with the network density and length distribution, as well as dipolar disorder-broadened $\left(\delta_{\text {dipole }}=5 \mathrm{meV}\right.$, width of the Gaussian distribution), we can simulate the measured charge density and temperature-dependent electronhole symmetric Seebeck coefficients within the Boltzmann transport formalism. As the transport energetics are strongly affected by the energy-dependent relaxation time, the Seebeck coefficient offers further insights into the carrier scattering mechanisms. Consequently, we can establish whether scattering in these networks can still be described as simple one-dimensional acoustic and optical phonon scattering as for single SWCNTs and discern the energy dependence of the relaxation time. ${ }^{22,31,32}$ The improved understanding of charge transport and energetics in SWCNT networks and their dependence on network parameters, allows us to provide design guidelines for both electronic and thermoelectric applications.

\section{Results and Discussion}

SWCNT Network Characterization. In order to study the impact of the chirality distribution on charge and thermoelectric transport in SWCNT networks, we selectively dispersed semiconducting SWCNTs from different growth processes in toluene using polymer wrapping with poly[(9,9-dioctylfluorenyl-2,7diyl)-alt-(6,6')-(2,2'-bipyridine) ] (PFO-BPy, molecular structure shown in Figure 1a). ${ }^{33,34}$ While using CoMoCAT raw nanotube material yielded an essentially monochiral dispersion of $(6,5)$ SWCNTs with a diameter of $0.76 \mathrm{~nm}$ and bandgap of $1.27 \mathrm{eV}$, using plasma torch grown SWCNTs resulted in a dispersion of a broader chirality distribution of large diameter (1.17-1.55 nm), small bandgap (0.70-0.88 eV) SWCNTs (RN SWCNTs). ${ }^{9,35}$ The diameter and bandgap range were determined from the $\mathrm{E}_{11}$ and $\mathrm{E}_{22}$ transitions in the UV-vis-NIR absorption spectra (Figure 1b). Via repeated washing of the SWCNT filter cakes with toluene $\left(80{ }^{\circ} \mathrm{C}\right.$, see Methods for further details) the amount of residual wrapping polymer in the SWCNTs networks became negligible (Figure 1b-f). Furthermore, the SWCNT networks were free of metallic nanotubes as evident from Raman spectroscopy (see Supporting Information Note 1). Both network density and length distribution of the networks were determined by atomic force microscopy (AFM) images (Figure 1c-f and Supporting Information Note 2). The length distribution depends on the raw material, the exfoliation method used for the polymer wrapping process as well as the re-dispersion method and conditions. ${ }^{35,36}$ The monochiral $(6,5)$ SWCNT networks have slightly longer tubes $(0.95 \pm 0.74 \mu \mathrm{m})$ than the RN networks $(0.57 \pm 0.29 \mu \mathrm{m})$. For a study of the intrinsic charge and thermoelectric transport properties of SWCNT networks it is important to consider relatively high network densities to reduce the impact of water or hydroxyl groups on polar surfaces that cause charge carrier trapping and hysteresis in transistor curves. ${ }^{37-39}$ Therefore, the impact of the network density on thermoelectric transport was investigated well above the percolation threshold and in the regime of mobility saturation (approx. $>13 \mu^{-1}$ ) by choosing a sparse network condition at the threshold of this regime and a dense network condition with at least twice the density. ${ }^{39}$ The AFM images of the $(6,5)$ and RN networks are shown in Figure 1c-f and the extracted network parameters are displayed in Table 1 . To probe the effect of small length variations we also investigated dense RN networks with shorter tubes (RN-dst, obtained from $60 \mathrm{~min}$ of re-dispersion, see Methods for further details). Since the $(6,5)$ networks showed a non-negligible trap density even for high network densities (evident from their stronger hysteresis in transistor transfer 


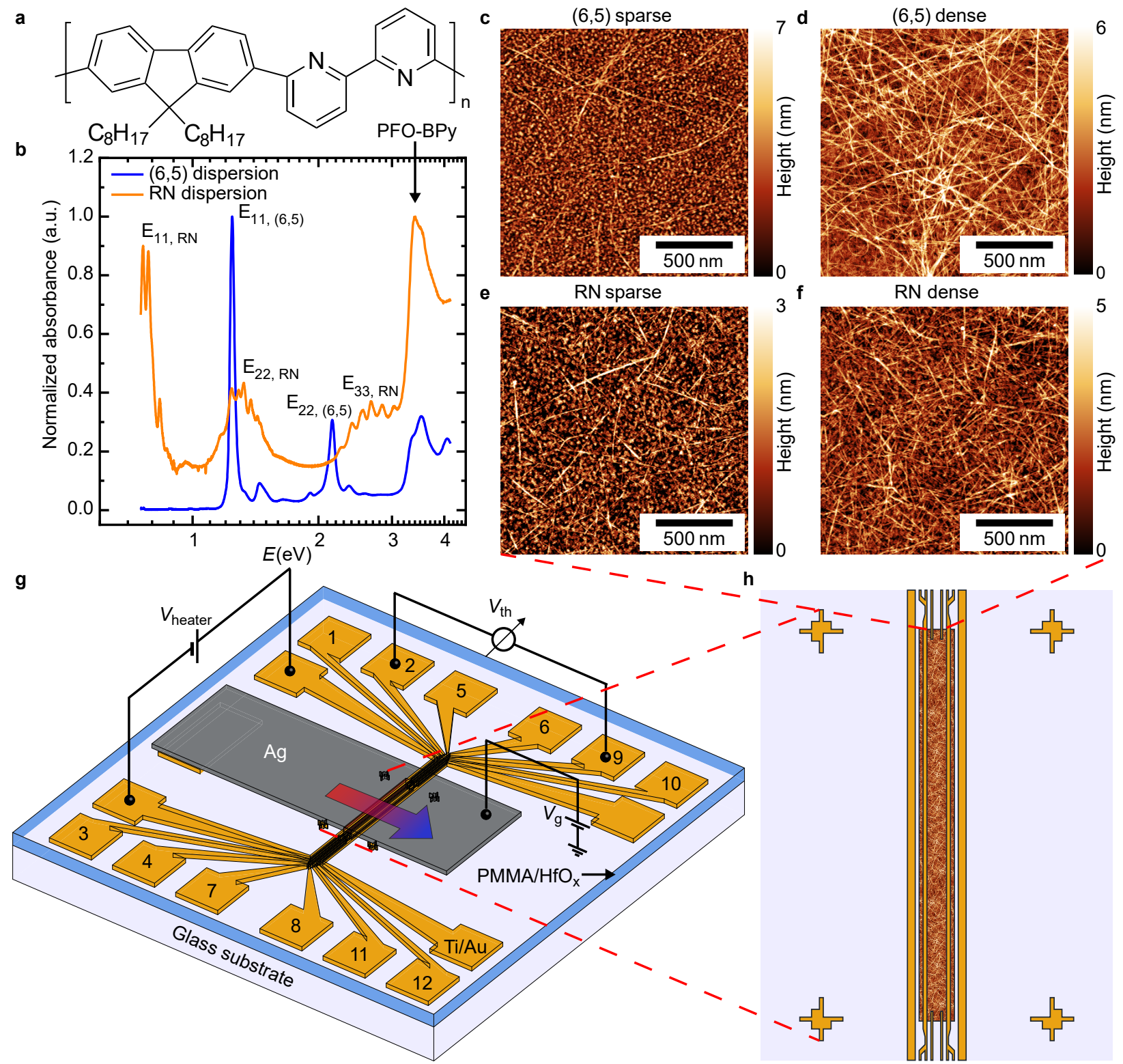

Figure 1. SWCNT network characterization and thermoelectric device architecture. The molecular structure of the wrapping polymer PFO-BPy (a), the normalized absorption spectra of the $(6,5)$ and RN dispersions (b), the AFM images of the $(6,5)$ sparse $(c),(6,5)$ dense (d), RN sparse (e) and RN dense (f) networks as well as the bottom-contact top-gate field-effect transistor architecture with four-pointprobe $(4 \mathrm{pp})$ contacts and two on-chip heaters $((\mathrm{g})$, close-up of the active area in $(\mathrm{h}))$ for the thermoelectric transport measurements are shown. In panel (g) pads 1-4 denote the source of the FET structure and the hot resistance sensor, pads 5-8 are 4pp voltage probes and pads 9-12 are the drain of the FET structure as well as the cold resistance sensor.

curves, larger onset voltages and stronger modulation of the Seebeck coefficient with charge density, see next section for further details), we also studied $(6,5)$ sparse networks treated with the strongly reducing ttmgb $((6,5)$-s-ttmgb), which blocks hole transport and removes residual water from the SWCNT network via protonation of ttmgb. ${ }^{24}$

To characterize the energetic disorder of the combined DoS and compare it with the thermoelectric transport energetics, photothermal deflection spectroscopy (PDS) measurements of the $(6,5)$ and RN networks were performed. PDS is a sensitive absorption measurement technique that detects heating due to nonradiative relaxation of the absorbed light. Due to its high sensitivity it is suitable for the detection of sub-bandgap tail states and the extraction of the Urbach energy $E_{\mathrm{u}}$, which is the characteristic energy of the exponential absorption tail in solid state absorption due to energetic disorder. ${ }^{40-42}$ In line with the tight-binding nearest-neighbour calculations and our thermoelectric transport measurements 
Table 1. SWCNT Network Parameters

\begin{tabular}{|c|c|c|c|}
\hline Network & Abbreviation & $\begin{array}{c}\text { Network } \\
\text { density }(\lambda) \\
\left(\mu \mathrm{m}^{-1}\right)\end{array}$ & $\begin{array}{c}\text { Average } \\
\text { SWCNT } \\
\text { length } \\
(\mu \mathrm{m})\end{array}$ \\
\hline $\begin{array}{l}(6,5) \text { sparse } \\
\text { (Figure 1c) }\end{array}$ & $(6,5)-\mathrm{s}$ & & \multirow{3}{*}{$0.95 \pm 0.74$} \\
\hline $\begin{array}{c}(6,5) \\
\text { sparse, } \\
\text { ttmgb- } \\
\text { treated }\end{array}$ & $\begin{array}{l}(6,5)-\mathrm{s}- \\
\text { ttmgb }\end{array}$ & $12.6 \pm 2.8$ & \\
\hline $\begin{array}{l}(6,5) \text { dense } \\
\text { (Figure 1d) }\end{array}$ & $(6,5)-\mathrm{d}$ & $31.7 \pm 3.1$ & \\
\hline $\begin{array}{l}\text { RN sparse, } \\
\text { long tubes } \\
\text { (Figure 1e) }\end{array}$ & RN-s-lt & $19.2 \pm 2.5$ & \multirow[b]{2}{*}{$0.57 \pm 0.29$} \\
\hline $\begin{array}{l}\text { RN dense, } \\
\text { long tubes } \\
\text { (Figure 1f) }\end{array}$ & RN-d-lt & $37.9 \pm 3.1$ & \\
\hline $\begin{array}{l}\text { RN dense, } \\
\text { short tubes }\end{array}$ & RN-d-st & $36.5 \pm 3.8$ & $0.36 \pm 0.20$ \\
\hline
\end{tabular}

we find a substantially smaller Urbach energy of $E_{\mathrm{u},(6,5)} \approx 30 \pm 3 \mathrm{meV}$ for the $(6,5)$ networks compared to $E_{\mathrm{u}, \mathrm{RN}} \approx 47 \pm 5 \mathrm{meV}$ for the $\mathrm{RN}$ networks (see Supporting Information Note 3). The larger energetic disorder of the bandtail of the RN networks originates from the close proximity in energy of the first van Hove singularities of representative chiralities $((16,2)$, $(10,9)$ and $(12,7)) \cdot{ }^{29,30,43}$

Electric and Thermoelectric Characterization at 300 K. Four-point-probe (4pp) linear mobility $\left(\mu_{4 \mathrm{pp}}\right)$, contact resistance $\left(R_{\mathrm{c}}\right)$, saturation mobility $\left(\mu_{\text {sat }}\right)$ as well as hole and electron Seebeck coefficient $\left(S_{\mathrm{h}}\right.$ and $\left.S_{\mathrm{e}}\right)$ of the six network conditions $((6,5)-\mathrm{s},(6,5)-\mathrm{d}$, (6,5)-s-ttmgb, RN-s-lt, RN-d-lt and RN-d-st) were characterized depending on temperature and charge density using a bottom-contact top-gate field-effect transistor (FET) architecture with 4pp voltage probes and two on-chip heaters (channel width $W=1000 \mu \mathrm{m}$ and length $L=50 \mu \mathrm{m}$, Figure $1 \mathrm{~g}, \mathrm{~h})$. The hybrid dielectric (60 nm high- $\kappa \mathrm{HfO}_{\mathrm{x}}$ on top of $11 \mathrm{~nm}$ poly(methyl methacrylate) (PMMA)) facilitated reduced dipolar disorder and lowvoltage operation with low gate leakage currents $(<100 \mathrm{pA}$, Figure $2 \mathrm{a}, \mathrm{b}) .{ }^{44}$ Source and drain electrodes were used as on-chip 4pp resistance thermometers for the temperature calibration of the Seebeck coefficient measurements (pads 1-4 and 9-12 in Figure 1g respectively, see Supporting Information Note 4 for further details). ${ }^{26,42}$ All untreated networks show balanced ambipolar transport characteristics with low hysteresis (onset voltage $\left(V_{\text {on }}\right)$ difference between forward and reverse sweep) and onset voltages $\left|V_{\text {on }}\right| \leq 2 \mathrm{~V}$ at $300 \mathrm{~K}$. The onset voltages are the voltages for which the drain-source current $\left(I_{\mathrm{ds}}\right)$ rises above the background noise and gate-leakage current level extracted from the linear transfer curves (see Supporting Information Note 5). The saturation transfer curves were measured in the condition $V_{\mathrm{ds}}=V_{\mathrm{gs}}-V_{\mathrm{on}}$ to ensure a unipolar saturation regime throughout the entire gate voltage range $(300 \mathrm{~K}$ measurements shown in Figure 2a, b). ${ }^{45}$ In the high network density regime (approx. $>13 \mu \mathrm{m}^{-1}$ ) both electric and thermoelectric transport are, within our measurement error of $5-10 \%$, independent of the network density (compare RN-s-lt and RN-dlt in Figure 2b, d) and small SWCNT length variations (compare $\mathrm{RN}-\mathrm{d}-\mathrm{lt}$ and $\mathrm{RN}-\mathrm{d}-\mathrm{st}$ in Figure 2b, d). ${ }^{39}$ Thermoelectric transport is electron-hole symmetric $\left(\left|S_{\mathrm{e}}\right| \approx S_{\mathrm{h}}\right)$ as expected from the electron-hole symmetric DoS for low energies in single SWCNTs (Figure 2c, d). ${ }^{29}$ The charge density was obtained from capacitance measurements on each device (see Supporting Information Note 5), using the relationship $n=\frac{C_{\mathrm{i}}}{h} \cdot\left(V_{\mathrm{gs}}-V_{\mathrm{on}}\right)$, with $C_{\mathrm{i}}$ being the areal capacitance and $h=2 \mathrm{~nm}$ the assumed height of the accumulation layer. ${ }^{41}$ Due to the absence of any metallic SWCNTs and the only moderately high charge densities, the magnitude of the Seebeck coefficient of all networks is several hundreds of $\frac{\mu \mathrm{V}}{\mathrm{K}}$ and decreases with increasing charge density. Particularly at high charge densities $\left(n>10^{19} \mathrm{~cm}^{-3}\right)$, the Seebeck coefficient of the monochiral $(6,5)$-s-ttmgb networks exceeds that of the mixed large diameter $\mathrm{RN}$ networks. The differences in the saturation transfer curves between the $(6,5)-\mathrm{d}$ and $(6,5)$-s networks originate from different trap state densities. Treatment with the electrondonating and highly alkaline ttmgb blocks hole transport and neutralizes electron trap states via protonation of ttmgb (molecular structure shown in inset of Figure 2a, see Supporting Information Note 5). ${ }^{24}$ The reduced trap den- 
a

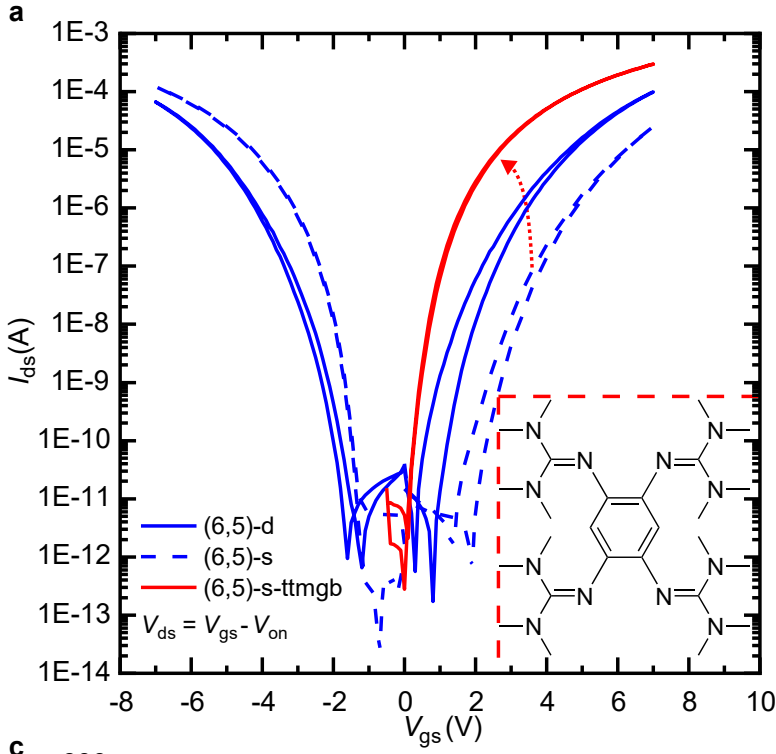

c

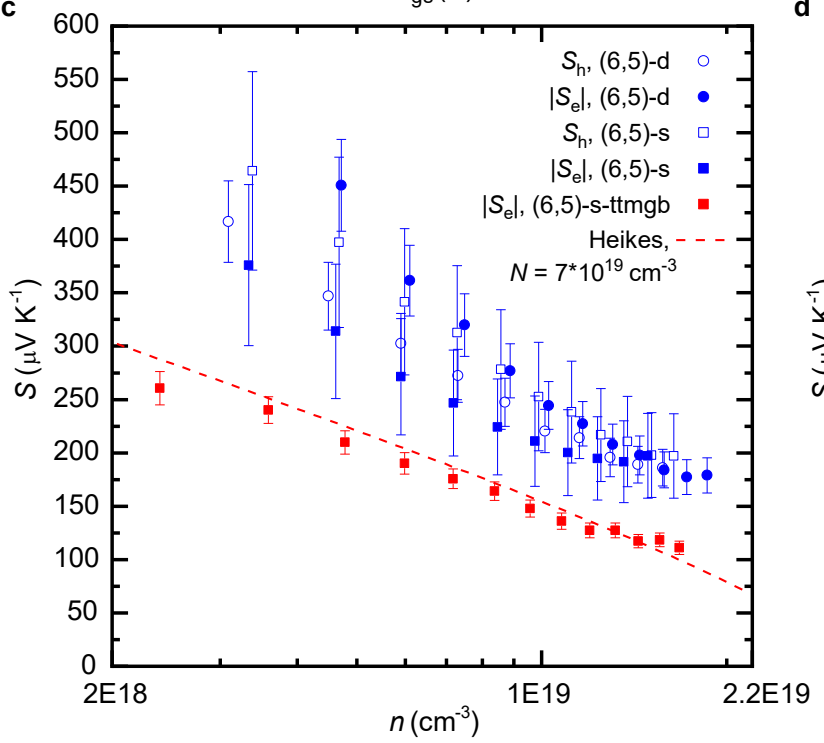

b
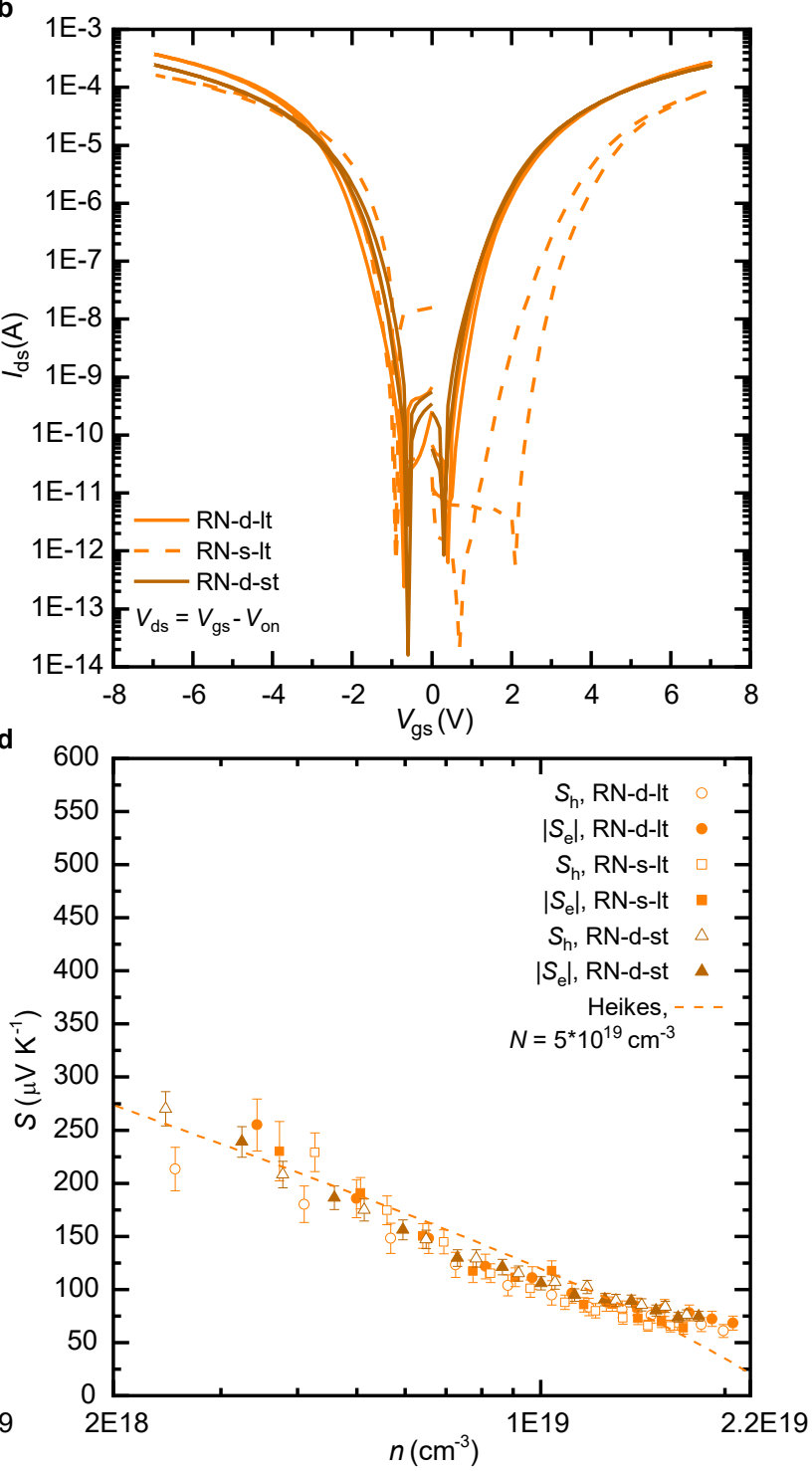

Figure 2. Charge and thermoelectric transport at $300 \mathrm{~K}$. Saturation transfer curves (with $V_{\mathrm{ds}}=V_{\mathrm{gs}}-V_{\mathrm{on}}$ ) as well as hole and absolute electron Seebeck coefficients $\left(S_{\mathrm{h}}\right.$ and $\left.\left|S_{\mathrm{e}}\right|\right)$ versus charge density of the $(6,5)$ dense $((6,5)$-d $),(6,5)$ sparse $((6,5)$-s $)$ and $(6,5)$ sparse ttmgbtreated ((6,5)-s-ttmgb) networks ((a) respectively (c)) as well as the long-tube RN dense (RN-d-lt), long-tube RN sparse (RN-s-lt) and short-tube RN dense (RN-d-st) networks ((b) respectively (d) are shown (network parameters in Table 1). The red arrow in (a) indicates the ttmgb treatment (molecular structure shown in inset of (a)). The red and orange dashed lines in (c) and (d) display Heikes formula with $N=7 \cdot 10^{19} \mathrm{~cm}^{-3}$ and $N=5 \cdot 10^{19} \mathrm{~cm}^{-3} .46$ The relative error in the Seebeck coefficient is $5-10 \%$ (except $20 \%$ for the $(6,5)$-s network), while the error analysis was performed according to Statz et al.. ${ }^{26}$

sity manifests itself through reduced hysteresis and onset voltage $\left(V_{\text {on, }(6,5)-\mathrm{s}, \mathrm{e}}=1.2 \mathrm{~V}\right.$ to $\left.V_{\text {on, }(6,5) \text {-s-ttmgb, e }}=0.0 \mathrm{~V}\right)$ as well as a decrease of the slope of the absolute electron Seebeck coefficient versus charge density (from approx. $251 \frac{\mu \mathrm{V}}{\mathrm{K} \cdot \mathrm{dec}}$ for the $(6,5)$-s networks to approx. $185 \frac{\mu \mathrm{V}}{\mathrm{K} \cdot \mathrm{dec}}$ for the $(6,5)$-s-ttmgb networks) due to reduced Fermi-level pinning by trap states. ${ }^{26}$ Heikes formula $\left(S=\frac{k_{\mathrm{B}}}{e} \ln \left(\frac{N-n}{n}\right)\right)$ for iso-energetic narrow-band conduction ( $N$ being the total thermally accessible DoS, $k_{\mathrm{B}}$ the Boltzmann constant and $e$ the electron charge) with $N=7 \cdot 10^{19} \mathrm{~cm}^{-3}$ and $N=5 \cdot 10^{19} \mathrm{~cm}^{-3}$ is shown in Figure 2c, d. ${ }^{46}$ At low charge densities Heikes formula describes the data well suggesting that in this regime $(n \ll N)$ the SWCNT networks behave as low-disorder systems due to the sharp DoS around the first van Hove singularity and the low dipolar disorder. As a result configurational entropy governs the Seebeck coefficient. However, for high charge densities the Seebeck coefficient of the SWCNT networks deviates from Heikes formula and the dependence of the Seebeck coefficient on carrier 
concentration becomes weaker. This also manifests itself in the slope of the Seebeck coefficient plotted versus the logarithm of carrier concentration and calculated over the whole range of data being slightly smaller than what is expected from Heikes formula $\left(198 \frac{\mu \mathrm{V}}{\mathrm{K} \cdot \mathrm{dec}}\right)$. The values are $185 \frac{\mu \mathrm{V}}{\mathrm{K} \cdot \mathrm{dec}}$ for the $(6,5)$-s-ttmgb and $188 \frac{\mu \mathrm{V}}{\mathrm{K} \cdot \mathrm{dec}}$ for the RN networks. The reduced carrier concentration dependence of the Seebeck coefficient is a manifestation of Boltzmann statistics becoming insufficient due to the Fermilevel $\left(E_{\mathrm{F}}\right)$ approaching the state distribution ( $n \ll N$ does not hold). In this regime a narrow-band description is inappropriate and the actual DoS of the networks needs to be considered (further details in section temperaturedependent thermoelectric transport in SWCNT networks). ${ }^{26}$

\section{Fluctuation-Induced Tunneling in}

SWCNT Networks. Electrical transport is thermally activated for all accessible charge densities (approx. $n \leq 2.2 \cdot 10^{19} \mathrm{~cm}^{-3}$ ) for both the $(6,5)$-s-ttmgb-treated and as previously reported for all untreated networks (Figure $3 \mathrm{a}, \mathrm{b}) .{ }^{9}$ Representative saturation electron mobilities $\left(\mu_{\mathrm{e}, \mathrm{sat}}\right)$ of the $(6,5)$-s-ttmgb and saturation hole mobilities $\left(\mu_{\mathrm{h}, \mathrm{sat}}\right)$ of the RN-d-st networks are shown in Figure 3a, b. 4pp linear and saturation mobilities are in good agreement (see Supporting Information Note 5) and all show a distinct maximum at $300 \mathrm{~K}$ within the charge density range of $n=1 \cdot 10^{18} \mathrm{~cm}^{-3}-2 \cdot 10^{19} \mathrm{~cm}^{-3}$ due to progressive filling of the first subband of the dipolar disorder-broadened one-dimensional DoS of the SWCNT networks with increasing charge density (Figure 3a, b) ${ }^{18-20}$ Due to the larger SWCNT diameters and the broader DoS originating from the presence of multiple chiralities in the RN networks, this filling effect occurs at lower charge densities than for the $(6,5)$ networks. The normalized saturation mobilities $\frac{\mu_{\text {sat }}}{\mu_{\mathrm{sat}, 300 \mathrm{~K}}}$ of the $(6,5)$-s-ttmgb network, extracted for seven different overdrive voltages $V_{\text {ov }}=V_{\text {gs }}-V_{\text {on }}(2$ to $5 \mathrm{~V}$ in steps of $0.5 \mathrm{~V}$ ) and hence charge densities $\left(4.8 \cdot 10^{18}\right.$ to $1.2 \cdot 10^{19} \mathrm{~cm}^{-3}$, line-cuts in Figure 3a) are plotted versus the inverse temperature in Figure 3c. Correspondingly, the normalized saturation mobilities of the RN-d-st network, extracted for six different overdrive voltages $(-2$ to $-4.5 \mathrm{~V}$ in steps of $-0.5 \mathrm{~V})$ and hence charge densities $\left(5.4 \cdot 10^{18}\right.$ to $1.2 \cdot 10^{19} \mathrm{~cm}^{-3}$, line-cuts in Figure $3 \mathrm{~b}$ ), are plotted versus the inverse temperature in Figure 3d. Despite the smaller energetic disorder experienced by carriers in the monochiral $(6,5)$ networks (temperature-dependent thermoelectric transport data in the following section) the charge density and temperature dependence of the saturation mobility for a fixed charge density (e.g. $\quad n_{0}=1.2 \cdot 10^{19} \mathrm{~cm}^{-3}$ ) and most notably for comparable DoS filling levels (e.g. $\quad n_{0}=1.2 \cdot 10^{19} \mathrm{~cm}^{-3}$ for $(6,5)$-sttmgb and $h_{0}=9.5 \cdot 10^{18} \mathrm{~cm}^{-3}$ for RN-d-st) of the $(6,5)$ networks is stronger than that of the RN networks as previously observed (Figure $3 \mathrm{c}, \mathrm{d}) .{ }^{47}$ This observation is not expected within a framework of a homogeneous medium and disorder-based transport rooted in variablerange or Miller-Abrahams hopping for which a lower disorder inevitably leads to a smaller charge density and temperature dependence of the field-effect mobility. ${ }^{26,48,49}$

We find that charge transport within semiconducting SWCNT networks can be described by the notions of transport in heterogeneous media and fluctuation-induced tunneling. ${ }^{9,11,27,50,51}$ We extend these general notions and emphasize that the heterogeneous medium for SWCNT networks consists of two main segments, the SWCNTs themselves with a Gaussian disorderbroadened, one-dimensional DoS that depends on the diameter distribution and the junctions with a distribution of tunnel barrier heights that depend on both the charge density, i.e. the position of the Fermi-level, as well as the diameter distribution of the constituting junctions. Transport along the SWCNTs is ballistic for short segments and even for long segments (longer than the mean free path due to acoustic phonon scattering) without SWCNT crossings, mobilities of $>1000 \frac{\mathrm{cm}^{2}}{\mathrm{~V} \cdot \mathrm{s}}$ can be achieved. ${ }^{22,23}$ While it has been shown that SWCNT crossings can lead to substantial reductions of the single SWCNT mobility, presumably due to enhanced scattering at these crossings, the con- 
a

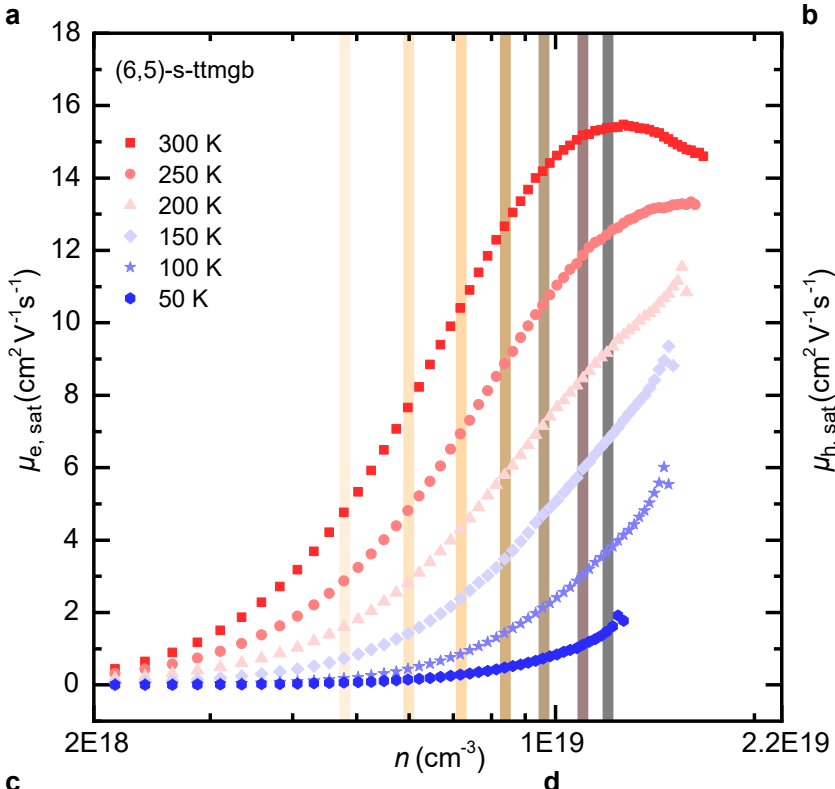

C

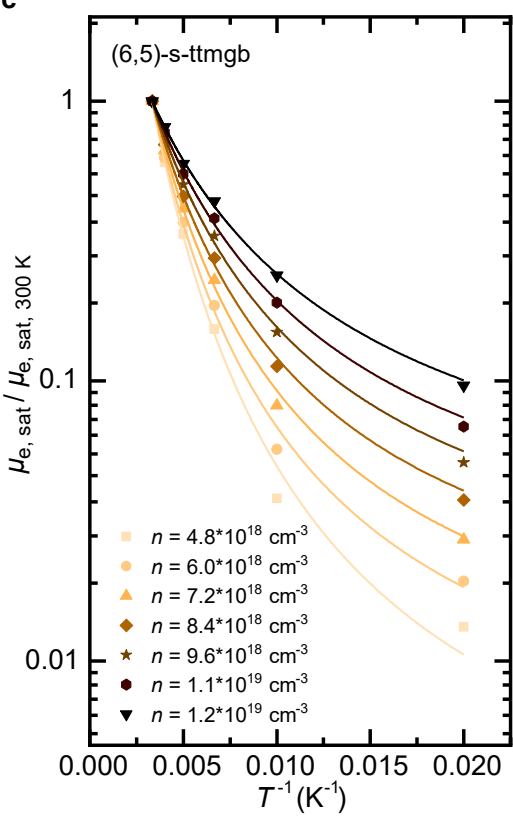

b

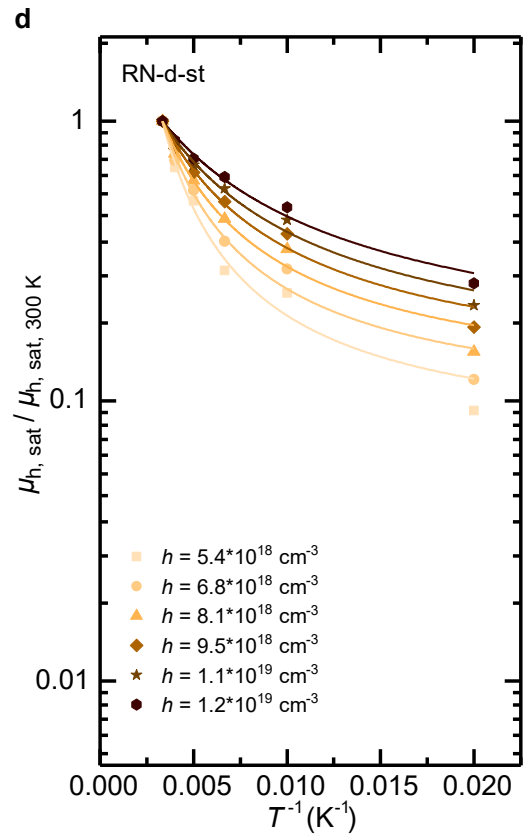

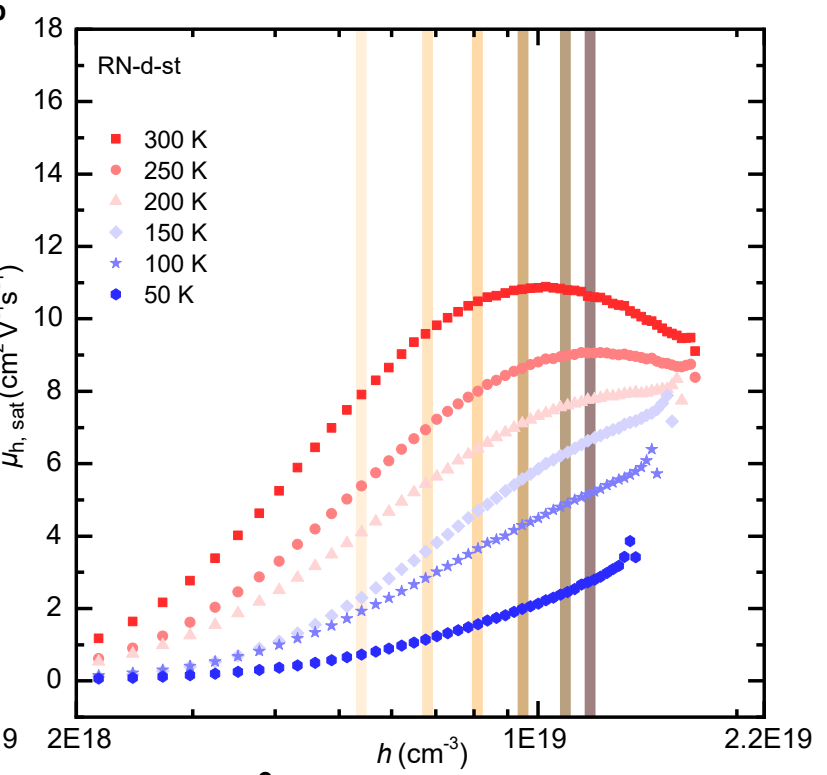

e

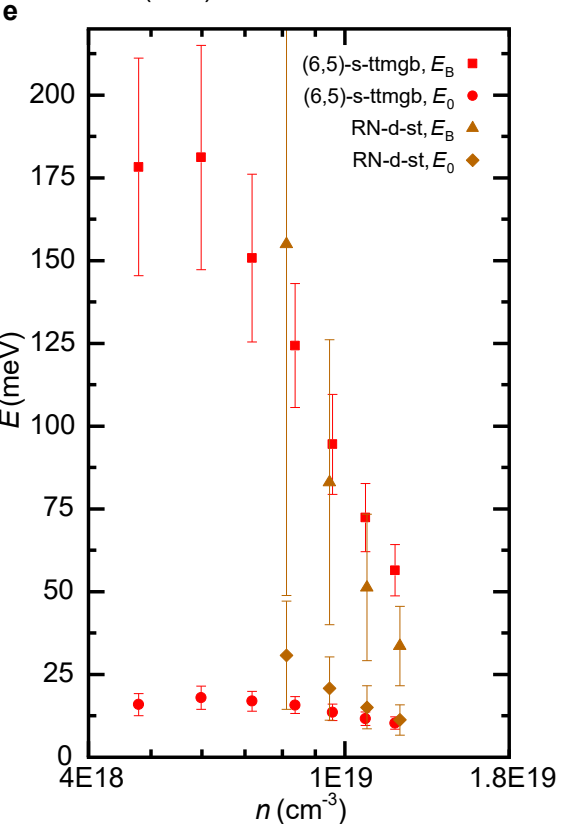

Figure 3. Fluctuation-induced tunneling (FIT) in SWCNT networks. The temperature and charge density-dependent electron saturation mobility $\left(\mu_{\mathrm{e}}\right.$, sat $)$ of the $(6,5)$-s-ttmgb network $(\mathrm{a}, \mathrm{c})$ and hole saturation mobility $\left(\mu_{\mathrm{h}, \text { sat }}\right)$ of the RN-d-st network (b, d) are shown. The normalized saturation mobilities $\frac{\mu_{\mathrm{sat}}}{\mu_{\mathrm{sat}, 300 \mathrm{~K}}}$ at several charge densities (line-cuts in (a) and (b)), and the FIT fits using the mobility analogue to eq 1 are shown in (c) and (d). The extracted characteristic energies $E_{\mathrm{B}}$ and $E_{0}$ are presented in (e). Error bars display the standard deviation of the fitting parameters.

ductivities of SWCNT segments will nevertheless be significantly larger than those of the tunnel barrier segments $\left(\sigma_{\mathrm{SWCNT}} \gg \sigma_{\mathrm{B}}\right) .{ }^{52,53}$ Within effective-medium theory the conductivity of each transport path $\left(\sigma_{j}\right)$ in the percolating network (with $N_{\text {path }}$ number of transport paths) can therefore be approximated with the conductivity of a single junction along this path for a peaked distribution of tunnel barrier heights. ${ }^{11,54,55}$ Transport through these tunnel barriers connecting two large conducting re- gions occurs through thermal activation across the tunnel barrier and fluctuation-induced tunneling, a thermally activated tunneling process due to voltage fluctuations across the junction capacitance caused by the random thermal motion of carriers (Johnson noise). Within this framework and the approximations of a parallel plate capacitance for the junction capacitance, a parabolic barrier approximation and the Wentzel-Kramers-Brillouin (WKB) approximation, the conductivity of SWCNT networks 
$(\sigma)$ can be written as: ${ }^{11}$

$$
\sigma \approx N_{\text {path }} \cdot \sigma_{B}=\sigma_{0}\left(E_{\mathrm{F}}\right) \cdot \exp \left(-\frac{T_{\mathrm{B}}\left(E_{\mathrm{F}}\right)}{T+T_{0}\left(E_{\mathrm{F}}\right)}\right)
$$

Here $T_{\mathrm{B}}\left(E_{\mathrm{F}}\right)=\frac{C \cdot\left(V_{\mathrm{B}}\left(E_{\mathrm{F}}\right)\right)^{2}}{2 \cdot k_{\mathrm{B}} \cdot e^{2}}$ and $T_{0}\left(E_{\mathrm{F}}\right)=$ $\frac{T_{\mathrm{B}}\left(E_{\mathrm{F}}\right)}{2 \cdot \chi \cdot w \cdot \xi(0)}$, while $C$ is the junction capacitance within the parallel plate approximation, $V_{\mathrm{B}}\left(E_{\mathrm{F}}\right)$ is the Fermi-level-dependent barrier height of the tunnel barrier, $k_{\mathrm{B}}$ is the Boltzmann constant, $e$ is the electron charge, $\chi=\left(\frac{2 \cdot m \cdot V_{\mathrm{B}}\left(E_{\mathrm{F}}\right)}{\hbar^{2}}\right)^{\frac{1}{2}}$ is the Fermi-level-dependent tunneling constant, $m$ is the effective carrier mass, $w$ is the width of the tunneling junction and $\xi(0)=\int_{0}^{1}(u \cdot(1-u))^{\frac{1}{2}} d u=\frac{\pi}{8}$ is a scaling factor that originates from the Taylor expansion of the transmission coefficient around the Fermi-level within the WKB approximation and depends on the parabolic shape of the tunnel barrier. $\sigma_{0}\left(E_{\mathrm{F}}\right)$ is a weakly temperaturedependent conductivity pre-factor that takes the number of conduction paths $N_{\text {path }}$ as well as the initial and final DoS on each side of the junction at the Fermi level $\left(g_{i}\left(E_{\mathrm{F}}\right)\right.$ and $\left.g_{f}\left(E_{\mathrm{F}}\right)\right)$ into account. $T_{\mathrm{B}}\left(E_{\mathrm{F}}\right)$ characterizes the temperature for which significant thermal excitation across the tunnel barrier is likely to occur and $T_{0}\left(E_{\mathrm{F}}\right)$ is the temperature above which thermal voltage fluctuations due to Johnson noise are relevant. For temperatures $T<T_{0}\left(E_{\mathrm{F}}\right)$ the expression becomes temperature-independent (elastic tunneling). The fact that there has not been any experimental evidence for an attainable charge density regime for which the conductivity in semiconducting SWCNT networks decreases with increasing temperature, further supports the validity of eq $1 .{ }^{10}$ The diameter dependence of the conductivity of the semiconducting SWCNT network within this framework is crucial. Firstly, the diameter distribution influences the energetic landscape (DoS) and thereby the position of the Fermilevel for a given charge density and temperature. The diameter distribution also influences the junction capacitance, since larger diameter SWCNTs are expected to have larger contact areas and therefore larger capacitances in the parallel plate approximation. Furthermore, smaller diameter tubes have been found to ex- hibit higher contact resistances, which has been attributed to higher tunnel barriers $V_{\mathrm{B}}\left(E_{\mathrm{F}}\right) .{ }^{53}$ Due to the existence of tunnel barriers for all charge densities, the temperature-activated behaviour of the saturation mobilities $(\sigma=$ $\left.\frac{C_{\mathrm{i}}}{h} \cdot \int_{V_{\mathrm{on}}}^{V_{\mathrm{gs}}} \mu_{\mathrm{sat}}\left(V_{\mathrm{gs}}^{\prime}\right) d V_{\mathrm{gs}}^{\prime}\right)$ within the framework of a heterogeneous medium and fluctuationinduced tunneling is expected (eq 1). The prefactor $\sigma_{0}\left(E_{\mathrm{F}}\right)$ accounts for the DoS filling effect and the existence of a mobility maximum caused by an eventual decrease of the initial and final DoS at the Fermi-level on each side of the junction $\left.\left(\sigma_{0}\left(E_{\mathrm{F}}\right) \propto g_{i}\left(E_{\mathrm{F}}\right), g_{f}\left(E_{\mathrm{F}}\right)\right)\right)$, which outweighs the slight reduction in the tunnel barrier height $V_{\mathrm{B}}\left(E_{\mathrm{F}}\right)$ in this charge density regime. Considering the proportionalities $T_{\mathrm{B}}\left(E_{\mathrm{F}}\right) \propto$ $C \cdot\left(V_{\mathrm{B}}\left(E_{\mathrm{F}}\right)\right)^{2}$ and $T_{0}\left(E_{\mathrm{F}}\right) \propto C \cdot\left(V_{\mathrm{B}}\left(E_{\mathrm{F}}\right)\right)^{\frac{3}{2}} \cdot m^{-\frac{1}{2}}$, the expected proportionality between junction capacitance and diameter as well as the antiproportionality between tunnel barrier height and diameter, ${ }^{53}$ the stronger charge density and temperature dependence of the saturation mobility of the $(6,5)$ compared to the RN networks (Figure 3a-d) may be rationalized, not as a manifestation of different degrees of disorder, but in terms of the dependence of the tunneling junctions between tubes on tube diameter: The smaller diameter of the $(6,5)$ SWCNTs is expected to lead to both higher tunnel barriers at any fixed charge density $\left(n_{0}\right)$ in the $(6,5)$ networks $\left(V_{\mathrm{B},(6,5)}\left(E_{\mathrm{F}}\left(n_{0}\right)\right)>V_{\mathrm{B}, \mathrm{RN}}\left(E_{\mathrm{F}}\left(n_{0}\right)\right)\right)$ as well as smaller SWCNT overlap areas resulting in smaller junction capacitances compared to the RN networks $\left(C_{(6,5)}<C_{\mathrm{RN}}\right) .{ }^{53}$ The characteristic energies of the saturation mobilities, $E_{\mathrm{B}}=k_{\mathrm{B}} T_{\mathrm{B}}$ and $E_{0}=k_{\mathrm{B}} T_{0}$, of the $(6,5)$-sttmgb and $\mathrm{RN}-\mathrm{d}$-st networks at various charge densities were obtained from fits of the normalized saturation mobilities $\frac{\mu_{\mathrm{sat}}}{\mu_{\mathrm{sat}, 300 \mathrm{~K}}}$ with an analogue to eq 1 (substituting $\sigma$ with $\mu_{\text {sat }}$, Figure $3 \mathrm{e})$. While we find higher characteristic barrier energies for the $(6,5)$ compared to the RN networks $\left(E_{\mathrm{B},(6,5)}\left(E_{\mathrm{F}}\right)>E_{\mathrm{B}, \mathrm{RN}}\left(E_{\mathrm{F}}\right)\right)$ for high charge densities (approx. $n \geq 10^{19} \mathrm{~cm}^{-3}$, Figure 3e), the distinction in the lower charge density regime is impeded by the high relative errors in the extraction of the characteristic energies. For the RN networks the character- 
istic energy of significant voltage fluctuations is larger $\left(E_{0, \mathrm{RN}}\left(E_{\mathrm{F}}\right)>E_{0,(6,5)}\left(E_{\mathrm{F}}\right)\right.$, Figure $\left.3 \mathrm{e}\right)$. This may reflect a smaller carrier effective mass and larger junction capacitance induced by the larger diameter of the RN tubes. Our experimental findings also confirm the expected slightly weaker charge density and Fermi-level dependence of $E_{\mathrm{B}}\left(E_{\mathrm{F}}\right)$ of the $(6,5)$ networks due to its narrower DoS (Figure 3e). We note that our assumption of a weakly temperaturedependent Fermi-level for the extraction of the characteristic energies can be justified by the observation of a temperature-independent Seebeck coefficient of the two networks within the temperature range of $150 \mathrm{~K}$ to $300 \mathrm{~K}$ and a weak temperature dependence below $150 \mathrm{~K}$ (within the measurement error of 5-10\%) due to the sharp DoS distribution of the networks (see following section).

Temperature-Dependent Thermoelectric Transport in SWCNT Networks. Based on the established independence of the Seebeck coefficient on network density and small SWCNT tube length variations in the high network density regime (approx. $>13 \mu \mathrm{m}^{-1}$ ) as well as its electron-hole symmetry (Figure $2 \mathrm{c}, \mathrm{d}$ ), we focus our temperature-dependent thermoelectric transport analysis on the absolute electron Seebeck coefficient $\left(\left|S_{\mathrm{e}}\right|\right)$ of the representative $(6,5)$-s-ttmgb and $\mathrm{RN}$-d-st networks (Figure 4). The full temperature dependence of the hole and absolute electron Seebeck coefficient of the six network conditions is presented in Supporting Information Note 6 . The Seebeck coefficients of the $(6,5)$-sttmgb and dense RN networks are temperatureindependent between $150 \mathrm{~K}$ and $300 \mathrm{~K}$ but show an increasing temperature dependence for lower temperatures (within the measurement error of $5-10 \%$, Figure 4b, d). The Seebeck coefficients of all untreated $(6,5)$ SWCNT networks and the sparse RN networks show a stronger and more gradual temperature dependence as well as a stronger charge density dependence due to higher trap densities and the resulting Fermi-level pinning (Figure 2c, Figure 4b, d and Supporting Information Note 6). ${ }^{26}$

We find that thermoelectric transport in semiconducting SWCNT networks can be modelled within the notions of transport in heterogeneous media and Boltzmann transport formalism. ${ }^{16,27}$ The relative orientation of transport path segments (SWCNTs and tunnel barriers) with respect to the temperature gradient has to be considered for the evaluation of the Seebeck coefficient. Kaiser pointed out that the tunnel barriers are likely to impact the thermal transport less than the electrical transport. ${ }^{12,27}$ Furthermore, in the percolating dense network density regime considered here, most tunnel barriers will be orthogonal to the temperature gradient and hence their contribution to the Seebeck coefficient is negligible. Therefore, the Seebeck coefficient of the SWCNT networks $(S)$ can be approximated as:

$$
S=\frac{\Delta T_{\mathrm{SWCNTs}}}{\Delta T} \cdot S_{\mathrm{SWCNTs}}+\frac{\Delta T_{\mathrm{B}}}{\Delta T} \cdot S_{\mathrm{B}} \approx S_{\mathrm{SWCNTS}}
$$

with $\Delta T_{\text {SWCNT }}$ and $S_{\text {SWCNTs }}$ being the temperature drop across the SWCNTs and their Seebeck coefficient, while $\Delta T_{\mathrm{B}}$ and $S_{\mathrm{B}}$ are the temperature drop across the tunnel barriers and their Seebeck coefficient, respectively. Consequently, we consider the Seebeck coefficient of the SWCNT networks to be dominated by the contributions to the Seebeck coefficient from the SWCNTs rather than the SWCNT junctions. In the presence of strong disorder potentials, e.g. when the charge density is introduced via nitrogen-substitution of carbon atoms in the SWCNTs, the contribution to the Seebeck coefficient by the SWCNTs has been proposed to be described by the Kubo rather than the Boltzmann transport formalism and by employing thermal Green's function formalism within a self-consistent $t$-matrix approximation. ${ }^{56}$ However, such strong disorder potentials are not present in our field-effectmodulated measurements of pristine SWCNT networks. Therefore, Boltzmann transport formalism is employed for the description of the Seebeck coefficient of the SWCNT networks. ${ }^{16}$ For the DoS of the SWCNT network $(g(E))$ we consider the convolution of the superposition of the DoS of the individual chiralities of the network $\left(g_{\mathrm{c}, i}(E)\right)$, weighted according to their relative fraction in the network $\left(w_{i}\right)$ and scaled according to the network den- 
a

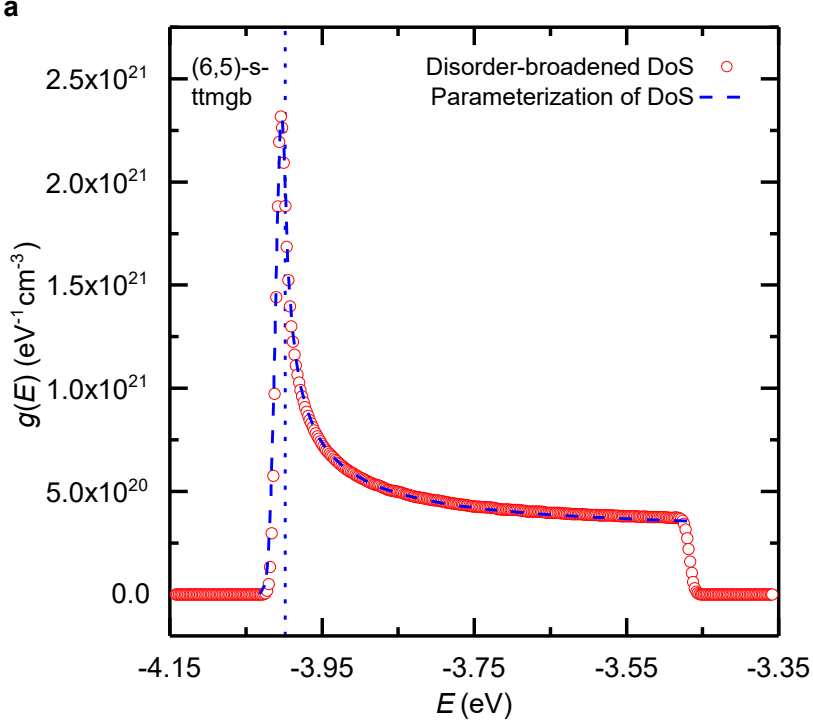

c

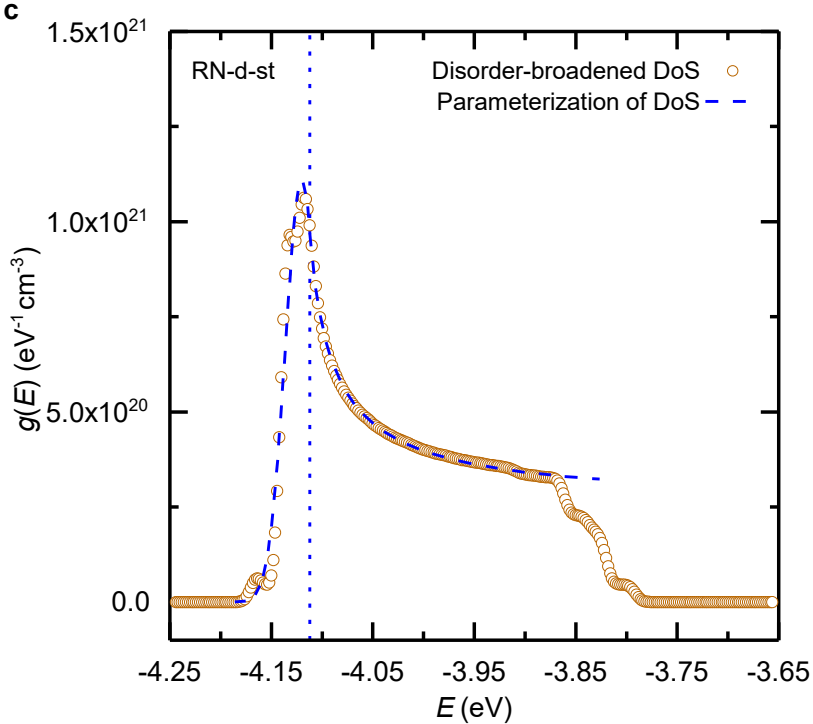

b

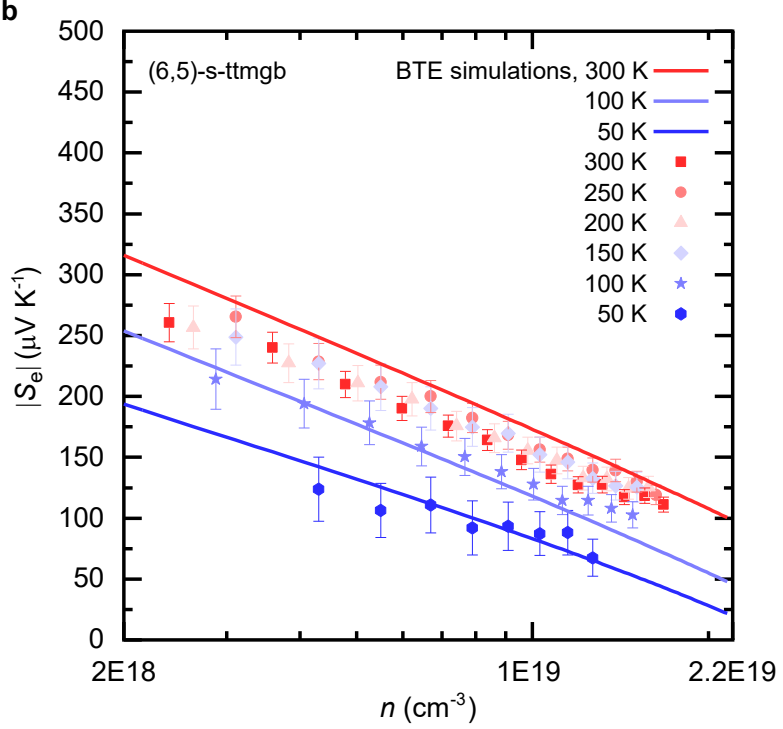

d

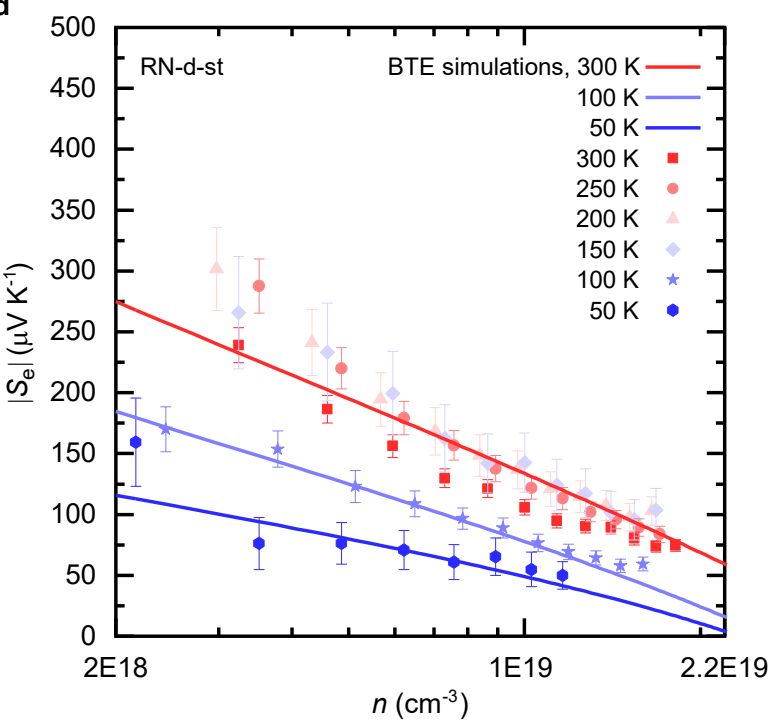

Figure 4. Temperature-dependent thermoelectric transport in SWCNT networks. The dipolar disorder-broadened $\left(\delta_{\text {dipole }}=5\right.$ meV $)$ DoS of the $(6,5)\left(\right.$ a) and RN (c) networks from tight-binding nearest-neighbour calculations including trigonal warping effects, ${ }^{29,30}$ scaled according to the network density and length distribution (Table 1) as well as the charge density and temperature-dependent absolute electron Seebeck coefficient $\left(\left|S_{\mathrm{e}}\right|\right)$ of the $(6,5)$-s-ttmgb (b) and RN-d-st (d) networks are shown. The blue dashed lines in panel (a) and (c) are the DoS parameterizations (Table 2) used for the BTE simulations of the Seebeck coefficients in panel (b) and (d). The vertical dotted blue lines in panel (a) and (c) indicate the transition energies from the disorder-based to the SWCNT-based parameterization. The relative error in the Seebeck coefficient is $5-10 \%$, while the error analysis was performed according to Statz et al.. ${ }^{26}$

sity and length distribution (Table 1), with a normalized Gaussian dipolar disorder distribution $\left(g_{\text {dipole }}(E)=\exp \left(-\frac{E^{2}}{2 \cdot \delta_{\text {dipole }}^{2}}\right)\right)$. For $g_{\mathrm{c}, i}(E)$ we consider reported tight-binding nearestneighbour (TBNN) calculations including trigonal warping effects. ${ }^{29,30}$ Given our charge density regime $\left(n \leq 2.2 \cdot 10^{19} \mathrm{~cm}^{-3}\right)$ and temperature range of $50 \mathrm{~K}$ to $300 \mathrm{~K}$ (low energies), it is sufficient to consider the first subband of the TBNN DoS of each chirality (further supported by the electrical data in Figure $3 a, b$ ). Therefore, the DoS of the SWCNT network can be expressed as:

$$
g(E)=N_{\mathrm{SWCNTs}} \cdot \frac{g_{\text {dipole }}(E) \circledast \sum_{i} w_{i} \cdot g_{c, i}(E)}{\int g_{\text {dipole }}(E) \circledast \sum_{i} w_{i} \cdot g_{c, i}(E) d E}
$$

where $N_{\mathrm{SWCNTS}}=\rho_{\mathrm{SWCNTS}} \cdot \bar{N}_{\text {single, SWCNT }}$ is the total first subband DoS of the SWCNTs in the network, $\rho_{\mathrm{SWCNTS}}=\beta \cdot \frac{\pi \cdot \lambda_{\mathrm{th}}}{2 \cdot \hat{l} \cdot \vec{d}}$ is the volumetric SWCNT density (interrelation assumes uniform random orientation of SWCNTs, see Supporting Information Note 7), ${ }^{18} \lambda_{\text {th }}$ the linear threshold network density for which the Seebeck coefficient becomes approximately independent of network density 
$\left(\sim 13 \mu \mathrm{m}^{-1}\right),{ }^{39} \tilde{l}$ and $\tilde{d}$ are the average SWCNT length and diameter of the SWCNTs in the network respectively, $\beta$ is a constant factor considering bundling of SWCNTs and $\bar{N}_{\text {single, } \mathrm{SWCNT}}=\int \sum_{i} w_{i} \cdot g_{\mathrm{c}, i}(E) d E$ is the average total DoS per single SWCNT.

Within the independent electron and relaxation time approximation the Seebeck coefficient can be derived from Boltzmann transport equation (BTE) and expressed as: ${ }^{16,25,57}$

$$
S=-\frac{\left\langle E-E_{\mathrm{F}}\right\rangle_{\sigma}}{e T}=-\frac{1}{e T}\left[\frac{\int\left(E-E_{\mathrm{F}}\right) \sigma(E) d E}{\int \sigma(E) d E}\right]
$$

where $\sigma(E)$ is the energy-dependent conductivity function in the expression for the conductivity:

$$
\begin{aligned}
\sigma & =\int \sigma(E) d E=\int \sigma^{\prime}(E)\left(-\frac{\partial f(E)}{\partial E}\right) d E \\
& =\int e^{2} v(E) \tau(E) v(E) g(E)\left(-\frac{\partial f(E)}{\partial E}\right) d E
\end{aligned}
$$

with $f(E)$ representing the Fermi-Dirac distribution function, $g(E)$ the DoS, $v(E)$ the carrier velocity and $\tau(E)$ the carrier relaxation time. Equation 4 emphasizes the physical meaning of the Seebeck coefficient as the entropy transported by a carrier divided by its charge and illustrates that each charge carrier contributes to the transported energy according to its excess energy with respect to the Fermi-level $\left(E-E_{\mathrm{F}}\right)$, weighted by the relative contribution of the conduction channel $\frac{\sigma(E)}{\sigma}$ to the total conductivity. ${ }^{25,26}$ With the same reasoning as for the DoS we can express the conductivity function without the occupancy factors of the SWCNT network $\left(\sigma^{\prime}(E)\right)$ as

$$
\sigma^{\prime}(E)=g_{\text {dipole }}(E) \circledast \sum_{i} w_{i} \cdot \sigma_{\mathrm{c}, i}^{\prime}(E)
$$

with $\sigma_{\mathrm{c}, i}^{\prime}(E)=v_{\mathrm{c}, i}(E)^{2} \tau_{\mathrm{c}, i}(E) g_{\mathrm{c}, i}(E)$. The carrier velocity within each SWCNT is determined by its dispersion relation $v_{\mathrm{c}, i}(E)=\frac{1}{\hbar}\left(\frac{\partial E}{\partial k}\right) \propto$ $g_{\mathrm{c}, i}(E)^{-1} \cdot{ }^{58}$ For the carrier relaxation time we investigate one-dimensional acoustic and optical phonon scattering $\tau_{\mathrm{c}, i}(E) \propto g_{\mathrm{c}, i}(E)^{-1}$ which is dominant in single SWCNTs as well as a more general power law relationship $\tau_{\mathrm{c}, i}(E) \propto$ $\left(E-E_{\mathrm{C}, i}\right)^{s}$, with $E_{\mathrm{C}, i}$ being the first van Hove singularity of the $i$-th chirality in the network and $s$ the scattering parameter characterizing the type of scattering. ${ }^{22,31,32,59}$

In order to simulate the Seebeck coefficient of the SWCNT networks using BTE, we parameterize both the TBNN DoS (eq 3) shown in Figure $4 \mathrm{a}, \mathrm{c}$ for the $(6,5)$ and $\mathrm{RN}$ networks as well as the conductivity function without occupancy factors $\left(\sigma^{\prime}(E)\right.$, see Supporting Information Note 7 ). The absolute energy scale in Figure 4a, c is based on the TBNN calculations as well as the redox potentials of the SWCNTs $\left(E_{\mathrm{F}_{0},(6,5)}=-4.55 \mathrm{eV}\right.$ for the $(6,5)$ networks and we assume $E_{\mathrm{F}_{0}, \mathrm{RN}} \approx-4.45 \mathrm{eV}$ for all chiralities in the RN network). ${ }^{43,60}$ For the RN network DoS we estimate a plausible chirality assignment based on an analysis of the absorption peaks $\left(w_{(17,3)}=0.04, w_{(16,2)}=0.28, w_{(10,9)}=\right.$ $0.2, w_{(12,7)}=0.12, w_{(13,5)}=0.22, w_{(10,8)}=$ 0.14 , see Supporting Information Note 7). The network DoS is parameterized (Figure 4a, c) with a disorder-dominated DoS for low energies $\left(E<E_{\text {trans }}, E_{\text {trans }}\right.$ being the transition energy, Figure 4a, c)

$$
g_{\mathrm{LE}}(E)=\frac{N_{\mathrm{C}}}{\sqrt{2 \pi \delta_{\mathrm{C}}}} \cdot \exp \left(-\frac{\left(E-E_{\mathrm{C}}\right)^{2}}{2 \delta_{\mathrm{C}}^{2}}\right)
$$

with $N_{\mathrm{C}}$ being the total DoS, $\delta_{\mathrm{C}}$ the width and $E_{\mathrm{C}}$ the center of the low energy DoS parameterization. For high energies $\left(E_{\text {trans }}<E<E_{\text {co }}\right.$, with $E_{\text {co }}$ being the cut-off energy of the parameterization) the network DoS is parameterized with a one-dimensional SWCNT DoS

$$
g_{\mathrm{HE}}(E)=N_{\mathrm{HE}} \cdot \frac{|E+| E_{\mathrm{F}_{0}}||}{\sqrt{\left(|E+| E_{\mathrm{F}_{0}}||\right)^{2}-\left(\left|E_{\mathrm{C}, \mathrm{HE}}+\right| E_{\mathrm{F}_{0}}||\right)^{2}}}
$$

with $N_{\mathrm{HE}}$ characterizing the magnitude of the DoS and $E_{\mathrm{C}, \mathrm{HE}}$ the energy of the first van Hove singularity of the high energy DoS parameterization. The parameters used for the parameterization of the TBNN network DoS that is employed for the Seebeck coefficient simulations in Figure 4b, d (solid lines) are summarized in Table 2. The only parameters in the BTE simulations of the Seebeck coefficients of the $(6,5)$ s-ttmgb and RN-d-st networks (Figure 4b,d) that are not a direct consequence of the network parameters and the TBNN DoS of the 
Table 2. Tight-Binding Nearest-Neighbour DoS Parameterization for Simulation of Seebeck Coefficients in SWCNT Networks.

\begin{tabular}{|c||c|c|c|c|c|c|c|c|}
\hline Network & $\begin{array}{c}\text { Total DoS } \\
\text { of the first } \\
\text { subband of } \\
\text { the } \\
\text { SWCNT } \\
\text { network } \\
\left(N_{\mathrm{SWCNTs}}\right)\end{array}$ & $\begin{array}{c}\text { Total DoS } \\
\text { of the low } \\
\text { energy DoS } \\
\left(N_{\mathrm{C}}\right)\end{array}$ & $\begin{array}{c}\text { Width of } \\
\text { the low } \\
\text { engy DoS } \\
\left(\delta_{\mathrm{C}}\right)\end{array}$ & $\begin{array}{c}\text { Center of } \\
\text { the low } \\
\text { energy DoS } \\
\left(E_{\mathrm{C}}\right)\end{array}$ & $\begin{array}{c}\text { Transition } \\
\text { energy } \\
\left(E_{\mathrm{trans}}\right)\end{array}$ & $\begin{array}{c}\text { Magnitude } \\
\text { of the high } \\
\text { energy DoS } \\
\left(N_{\mathrm{HE}}\right)\end{array}$ & $\begin{array}{c}\text { First van } \\
\text { Hove } \\
\text { singularity } \\
\text { of the high } \\
\text { energy DoS } \\
\left(E_{\mathrm{HE}}\right)\end{array}$ & $\begin{array}{c}\text { Cut-off } \\
\text { energy } \\
\left(E_{\mathrm{co}}\right)\end{array}$ \\
\begin{tabular}{c|c|c|c|c|c|}
$(6,5)-\mathrm{s}-$ \\
tmgb \\
$($ Figure $4 \mathrm{a})$
\end{tabular} & $\begin{array}{c}2.94 \cdot 10^{20} \\
\mathrm{~cm}^{-3}\end{array}$ & $\begin{array}{c}4.66 \cdot 10^{19} \\
\mathrm{~cm}^{-3}\end{array}$ & $8.1 \mathrm{meV}$ & $-4.003 \mathrm{eV}$ & $-3.998 \mathrm{eV}$ & $\begin{array}{c}3.080 \cdot 10^{20} \\
\mathrm{~cm}^{-3} \mathrm{eV}^{-1}\end{array}$ & $-4.005 \mathrm{eV}$ & $-3.467 \mathrm{eV}$ \\
\hline $\begin{array}{c}\text { RN-d-st } \\
(\text { Figure 4c) }\end{array}$ & $\begin{array}{c}1.50 \cdot 10^{20} \\
\mathrm{~cm}^{-3}\end{array}$ & $\begin{array}{c}4.49 \cdot 10^{19} \\
\mathrm{~cm}^{-3}\end{array}$ & $16.2 \mathrm{meV}$ & $-4.120 \mathrm{eV}$ & $-4.112 \mathrm{eV}$ & $\begin{array}{c}2.760 \cdot 10^{20} \\
\mathrm{~cm}^{-3} \mathrm{eV}^{-1}\end{array}$ & $-4.126 \mathrm{eV}$ & $-3.826 \mathrm{eV}$ \\
\hline
\end{tabular}

SWCNTs, are the width of the disorder distribution $\left(\delta_{\text {dipole }}=5 \mathrm{meV}\right)$ and the scattering parameter $s=-1$, which are identical for the two networks, as well as the bundling factor $\beta$ that accounts for bundling of SWCNTs in the network $\left(\beta_{(6,5)} \approx 2.9\right.$ and $\left.\beta_{\mathrm{RN}} \approx 4.3\right)$. In Supporting Information Note 7 we show that one-dimensional acoustic and optical phonon scattering with $\tau_{\mathrm{c}, i}(E) \propto g_{\mathrm{c}, i}(E)^{-1}$ cannot explain the charge density and temperature dependence of the Seebeck coefficient. This form of scattering would give too much weight to the high energy conduction channels, resulting in a strongly temperature-dependent thermally accessible DoS, which would lead to a much stronger temperature dependence of the Seebeck coefficient between $100 \mathrm{~K}$ and $300 \mathrm{~K}$ than observed experimentally (see Figure $4 \mathrm{~b}$ and Supporting Information Note 7). Instead we find that a good fit of the temperaturedependent Seebeck coefficient requires the scattering parameter $s$ to be approximately -1 . Our BTE simulations based on the dipolar disorder-broadened TBNN DoS allow insights into the microscopic transport mechanisms as well as the origins of the charge density and temperature dependence of the Seebeck coefficients of the SWCNT networks. The magnitude of the Seebeck coefficient primarily depends on the magnitude and sharpness of the DoS within the energy window $f(E) \cdot[1-f(E)]$ which determines the states participating in transport. Since the $(6,5)$ SWCNTs have more states in the vicinity of the first van Hove singularity than the large diameter SWCNTs present in the RN networks, the low energy DoS of the
$(6,5)$ networks, which dominates transport in our charge density regime, is higher than that of the RN networks $\left(N_{\mathrm{C},(6,5)}>N_{\mathrm{C}, \mathrm{RN}}\right.$, see Table 2). The latter is the dominant factor for the observed higher Seebeck coefficients (Figure $4 \mathrm{~b}, \mathrm{~d}$ ). As expected from the mono-chirality of the $(6,5)$ network and in agreement with our PDS measurements $\left(E_{\mathrm{u},(6,5)} \approx 30 \pm 3 \mathrm{meV}\right.$ and $\left.E_{\mathrm{u}, \mathrm{RN}} \approx 47 \pm 5 \mathrm{meV}\right)$, the low energy $(6,5) \mathrm{DoS}$ is effectively much sharper than that of the RN networks $\left(\delta_{\mathrm{C},(6,5)} \approx 8.1 \mathrm{meV}\right.$ versus $\delta_{\mathrm{C}, \mathrm{RN}} \approx$ $16.2 \mathrm{meV}$ ). This in turn leads to the weaker temperature dependence of the Seebeck coefficient of the $(6,5)$ compared to the RN networks (Figure 4b, d). In addition, the sharper DoS in combination with the energy dependence of the relaxation time $\left(\tau_{\mathrm{c}, i}(E) \propto\left(E-E_{\mathrm{C}, i}\right)^{s}\right.$, $s=-1$ ), leads to a stronger charge density dependence of the Seebeck coefficient in the $(6,5)$ networks at lower temperatures, because the dominant transport energies are pinned energetically closer to the van Hove singularity (Figure 4b, d). The energy dependence of the relaxation time with $s=-1$ is different from that of individual SWCNTs. This is likely to reflect energy-dependent relaxation processes associated with the tube junctions or it might be a manifestation that the effective dimensionality of the SWCNT networks is not one-, but two-dimensional. We note that the energy dependence of the relaxation time due to acoustic phonon scattering in graphene is also characterized by $s=-1 .{ }^{61,62}$ This observation may warrant a detailed theoretical study that takes into account an appropriate deformation potential and scattering at the SWCNT junctions. 
Implications for SWCNT Network FETs and Thermoelectics. Based on our electrical transport data (Figure 3 and inset of Figure 5), two important, general guidelines for SWCNT network FETs are evident in our measurements. Firstly, even SWCNT networks with ambi-

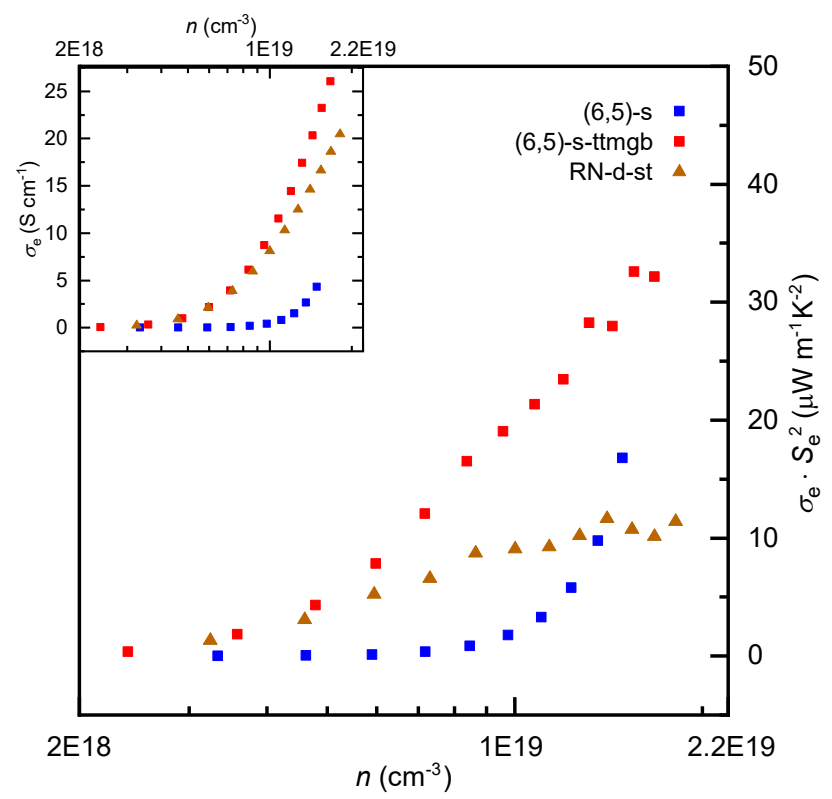

Figure 5. Thermoelectric performance at $300 \mathrm{~K}$. The charge density-dependent electron power factors $\left(\sigma_{\mathrm{e}} \cdot S_{\mathrm{e}}^{2}\right)$ and conductivities (inset) of the $(6,5)$-s, $(6,5)$-s-ttmgb and RN-d-st networks are shown. The monochiral $(6,5)$-s-ttmgb networks strongly outperform the broad diameter distribution RN networks due to their higher Seebeck coefficients (Figure 2c, d), emphasizing both the importance of trap state minimization as well as chirality selection to narrow and increase the thermally accessible DoS.

polar transport character and moderate hysteresis can still exhibit significant amount of trap states $((6,5)$ networks, Figure 2a). Minimizing this trap state density by treatments such as the ttmgb-treatment used here is essential for further performance optimization of FETs based on SWCNT networks. ${ }^{24}$ Secondly, large diameter $(1.2-1.6 \mathrm{~nm})$ SWCNT networks show higher carrier mobilites at comparable trap densities probably due to lower tunnel barriers. Chirality selection towards monochiral networks enables enhanced mobilities due to reduced energetic disorder via a narrower thermally accessible DoS. ${ }^{47,53}$ The upper diameter limit is given by the on/off current ratio requirements of the electronic application. Consequently, we suggest trap density-minimized monochiral SWCNTs of large diameter in the range of 1.2-1.6 nm, such as the recently demonstrated
$(14,6)$ networks, for high on/off current ratio, high-mobility SWCNT network FETs. ${ }^{47,63}$

The conversion efficiency of waste heat to electricity by thermoelectric materials is governed by the figure of merit $z T=\frac{P F}{\kappa} \cdot T=\frac{\sigma \cdot S^{2}}{\kappa} \cdot T$, with the power factor $P F=\sigma \cdot S^{2}, \sigma$ the electrical conductivity, $S$ the Seebeck coefficient, $\kappa$ the thermal conductivity and $T$ the temperature. ${ }^{64}$ Highly doped SWCNT networks have displayed ambipolar power factors in excess of $500 \frac{\mu \mathrm{W}}{\mathrm{m} \cdot \mathrm{K}^{2}}$ and are therefore promising candidates for next generation flexible thermoelectric generators. ${ }^{65}$ For further improvements in device performance the optimal chirality distribution of the SWCNT networks needs to be established. ${ }^{4,66}$ We therefore present the charge density-dependent electron power factors $\left(\sigma_{\mathrm{e}}\right.$. $\left.S_{\mathrm{e}}^{2}\right)$ of the $(6,5)$-s, $(6,5)$-s-ttmgb and RN-d-st networks at $300 \mathrm{~K}$ in Figure 5. Despite the significantly lower conductivities of the $(6,5)$ networks (inset of Figure 5), the power factors of the $(6,5)$ networks exceed those of the RN networks, particularly for high charge densities relevant for applications. The power factors of the trap-free ttmgb-treated $(6,5)$ networks exceed those of the RN networks for almost all investigated charge densities. As revealed by our BTE simulations, this is caused by the higher Seebeck coefficients primarily due to the larger DoS of the $(6,5)$ SWCNTs in the vicinity of the first van Hove singularity $\left(N_{\mathrm{C},(6,5)}>N_{\mathrm{C}, \mathrm{RN}}\right.$, see Table 2). For practical applications the charge density needs to be tuned to higher levels on the order of several times $10^{20} \mathrm{~cm}^{-3}$, allowing the Fermi-level to be in the vicinity of the second van Hove singularity. ${ }^{20,65,66}$ This will be the optimal Fermi-level position, as it allows for both high conductivities as well as a large thermally accessible DoS within the transport window $f(E) \cdot[1-f(E)]$ leading to high Seebeck coefficients. In terms of the Seebeck coefficient multiple chiralities in the network are acceptable if the effective width of the network DoS around the second van Hove singularities is still narrower than the transport window $\left(\sim 4 k_{\mathrm{B}} T\right)$, as it would allow for a comparable thermally accessible DoS (with only slightly reduced Seebeck coefficient due to the Fermi-level being closer to the van Hove singularity). Neverthe- 
less, given that the conductivities of monochiral networks are expected to be higher, as well as the fact that molecular doping introduces additional energetic disorder, monochiral networks are still preferable. Our simulations also indicate that the effect of smaller diameter tubes having a larger thermally accessible DoS in the vicinity of the van Hove singularities will still prevail, meaning that the optimal diameter for SWCNT network based thermoelectric devices will likely be smaller than that for FETs, where the reduced Seebeck coefficient of larger diameter tubes is no constraint. The precise optimal diameter can only be predicted once more data on trap-free monochiral networks is available. Therefore, in order to benefit from both lower tunnel barriers and higher conductivities, as well as high Seebeck coefficients, we propose to investigate a series of moderately large (1.0$1.4 \mathrm{~nm})$ monochiral networks, such as the recently demonstrated $(14,6)$ networks. ${ }^{63}$

\section{Conclusions}

In summary, we have shown that charge and thermoelectric transport in polymer-sorted SWCNT networks with certain diameters and different network densities as well as length distributions, can be modelled by the Boltzmann transport formalism, incorporating transport in heterogeneous media and fluctuation-induced tunneling. We directly relate the charge density and temperature dependence of the Seebeck coefficient in these networks to their dipolar disorder-broadened and network parameterscaled TBNN DoS. Furthermore, our BTE simulations of the Seebeck coefficients point towards a more two-dimensional character of carrier scattering and the necessity to consider scattering at SWCNT junctions as the relaxation time is found to be anti-proportional to the energy in these SWCNT networks, as opposed to simple one-dimensional acoustic and optical phonon scattering found in single SWCNTs. Based on our fundamental insights on charge and thermoelectric transport, we propose narrow DoS distribution (ideally monochiral), large diameter SWCNT networks, that enable a large thermally accessible DoS leading to high Seebeck coefficients as well as low tunnel barriers and high conductivities for both electronic and thermoelectric applications. While for electronic applications the on/off current ratio poses an upper limit for the SWCNT diameter $(1.2-1.6 \mathrm{~nm})$, the additional requirement of high Seebeck coefficients in thermoelectric devices is likely to be a stronger constraint on the SWCNT diameter (1.0-1.4 nm).

\section{Methods}

Preparation of SWCNT Dispersions. As described previously, semiconducting SWCNTs were selected from CoMoCAT (CHASM Advanced Materials Inc., SG65i-L58, diameter 0.7-1.0 nm) and plasma torch (Raymor Industries Inc., RN-220, diameter 0.9-1.5 nm, batch RNB739-220-A329) raw material by polymer wrapping in toluene (analytical grade, VWR Chemicals) using poly[(9,9-dioctylfluorenyl2,7-diyl)-alt-(6,6')-(2,2'-bipyridine)] (PFOBPy, American Dye Source Inc., ADS153UV, $\left.M_{\mathrm{W}}=40 \mathrm{~kg} \mathrm{~mol}^{-1}\right) \cdot{ }^{35,47}(6,5)$ SWCNT dispersions were prepared by adding CoMoCAT $\left(0.38 \mathrm{~g} \mathrm{~L}^{-1}\right)$ raw material to a solution of PFOBPy $\left(0.5 \mathrm{~g} \mathrm{~L}^{-1}\right)$ in toluene, and shear-forcemixing (Silverson L2/Air, $10230 \mathrm{rpm}$ ) for $72 \mathrm{~h}$ at $20{ }^{\circ} \mathrm{C}$ with two subsequent centrifugation steps at $60000 \mathrm{~g}$ (Beckman Coulter Avanti J26XP) for $45 \mathrm{~min}$. The supernatant containing the wrapped $(6,5)$ SWCNTs was collected after each step. Mixed SWCNTs were selected by adding plasma torch raw material $\left(1.5 \mathrm{~g} \mathrm{~L}^{-1}\right)$ to a PFO-BPy/toluene solution $\left(0.5 \mathrm{~g} \mathrm{~L}^{-1}\right)$ and bath sonication for $60 \mathrm{~min}$ at $20{ }^{\circ} \mathrm{C}$ followed by centrifugation as described above. All dispersions were passed through PTFE membrane filters (Merck Millipore, JVWP, $0.1 \mu \mathrm{m}$ pore size) and the resulting $(6,5)$ and mixed SWCNT filter cakes were washed with toluene $\left(80{ }^{\circ} \mathrm{C}\right.$, three times). The filter cake was redispersed in pure toluene $(1 \mathrm{ml})$ by bath sonication for $30 \mathrm{~min}$ at $20{ }^{\circ} \mathrm{C}$.

\section{Dopant Synthesis.}

1,2,4,5-Tetrakis(tetramethylguanidino)benzene (ttmgb) was synthesized and purified as de- 
scribed previously and the colourless crystals were subsequently stored in dry nitrogen. ${ }^{67,68}$ Solutions of ttmgb in anhydrous toluene were prepared directly prior to use.

Device Preparation. The device architecture, a bottom-contact top-gate field-effect transistor structure with four-point-voltage probes and two on-chip heaters is shown in Figure 1g. The electrode design was patterned on AF 32 eco glass (Schott AG) by standard photolithography in combination with electronbeam evaporation of titanium $(3 \mathrm{~nm})$ and gold (12 nm) with subsequent lift-off in $N$-methyl2-pyrrolidone (HPLC grade, Sigma). SWCNT dispersions were spin-coated at $2000 \mathrm{rpm}$ for $30 \mathrm{~s}$ and annealed at $100{ }^{\circ} \mathrm{C}$ (three times). After thorough rinsing with tetrahydrofuran and isopropyl alcohol to remove residual excess polymer, the films were patterned with standard photolithography and oxygen plasma etching. The substrates were annealed at $300{ }^{\circ} \mathrm{C}$ for $30 \mathrm{~min}$ in dry nitrogen; ttmgb $\left(2.5 \mathrm{~g} \mathrm{~L}^{-1}\right)$ was applied to selected samples by dip-coating in a solution in anhydrous toluene for $20 \mathrm{~min}$ and subsequent annealing at $150{ }^{\circ} \mathrm{C}$ for $30 \mathrm{~min}$. Spin-coating of PMMA (syndiotactic poly(methyl methacrylate), Polymer Source, $M_{\mathrm{W}}=315 \mathrm{~kg} \mathrm{~mol}^{-1}, 6 \mathrm{~g} \mathrm{~L}^{-1}$ ) in $n$ butyl acetate at $4000 \mathrm{rpm}$ for $60 \mathrm{~s}$ and atomic layer deposition of $\mathrm{HfO}_{\mathrm{x}}$ (Ultratech Savannah S100) at $100{ }^{\circ} \mathrm{C}$ with water as the oxygen source and tetrakis(dimethylamino)hafnium (Strem Chemicals Inc.) as the precursor formed the hybrid dielectric (PMMA (11 nm), $\mathrm{HfO}_{\mathrm{x}}$ $(60 \mathrm{~nm})) .{ }^{44}$ The devices were completed by thermal evaporation of silver gate electrodes (30 nm).

SWCNT Network Characterization. Absorption spectra of the SWCNT dispersions were obtained with a Cary 6000i absorption spectrometer (Varian Inc.). Raman spectra of the deposited SWCNT networks were recorded with a Renishaw plc inVia Reflex confocal Raman microscope with a 50x objective (Leica, $\mathrm{NA}=0.75$ ). Maps consisting of approximately 1600 spectra over an area of $100 \times 100 \mu^{2}$ were collected in StreamLine mode with three different lasers (532 nm, $633 \mathrm{~nm}$ and $785 \mathrm{~nm}$ ). For each sample, the spectra were averaged and baseline-corrected. Atomic force microscopy images were collected using a Bruker corp. Dimension Icon atomic force microscope in ScanAsyst mode. Photothermal deflection spectroscopy (PDS) measurements were performed with a custom setup similar to the one presented by Jackson and coworkers. ${ }^{42,69}$ Apart from using Heraeus Spectrosil 2000 substrates, the SWCNT networks were prepared as for the thermoelectric devices. The samples were held in a hermetically sealed Suprasil quartz cuvette (Hellma GmbH \& Co. KG) filled with an inert liquid (3M Fluorinert Electronic Liquid FC-72), excited with a modulated monochromated light beam produced by a $100 \mathrm{~W}$ Xenon arc lamp (Osram GmbH) and a CVI Digikrom DK240 monochromator and probed with a $670 \mathrm{~nm}$ fibre-coupled diode laser (iFLEX2000, Qioptiq Ltd.). The beam deflections were measured using a quadrant photodiode and a lock-in amplifier (Stanford Research Systems SR830). The data and extracted Urbach energies are presented in Supporting Information Note 3.

SWCNT Device Characterization. Transistor transfer and output, sensor resistance calibration and thermal voltage measurements were performed under vacuum $\left(<10^{-6}\right.$ mbar $)$ in a six-probe closed-cycle cryogenic probe station (Lake Shore Cryotronics Inc., CRX$4 \mathrm{~K})$ with two Keithley 2612B Dual Channel SourceMeters and one Keithley 6430 SubFemtoamp Remote SourceMeter including Remote PreAmp. While the principle of the sensor resistance calibration and thermal voltage measurements is the same as in our previous studies, ${ }^{26,42}$ we have further improved the unidirectionality of the temperature gradient and the accuracy of the on-chip resistive thermometers. The details of the measurement configuration are presented in Supporting Information Note 4 . The device capacitance was determined using an impedance spectrometer (ModuLab XM MTS System, Solartron Analytical). Measurements were conducted at $1.0 \mathrm{kHz}$, and the maximum capacitance $C_{\mathrm{m}}$ was extracted in the on-state $\left(V_{\mathrm{gs}}= \pm 5 \mathrm{~V}\right.$, for electron and hole transport respectively). Consequently, the areal capacitance $C_{\mathrm{i}}=\frac{C_{\mathrm{m}}}{W \cdot L_{\mathrm{pattern}}}$ $\left(L_{\text {pattern }}=90 \mu \mathrm{m}\right.$ being the entire length of the 
SWCNT patterned area) was obtained. We determined both four-point-probe linear $\left(\mu_{4 \mathrm{pp}}\right)$ as well as saturation mobilities $\left(\mu_{\text {sat }}\right)$. The fourpoint-probe linear mobilities and contact resistances were obtained at $V_{\mathrm{ds}}=-0.1 \mathrm{~V}$ (apart from $V_{\mathrm{ds}}=+0.1 \mathrm{~V}$ for the $n$-type $(6,5)$-s-ttmgb device) using two $5 \mu \mathrm{m}$ wide voltage probes located at $L_{\mathrm{P} 1}=12.5 \mu \mathrm{m}$ and $L_{\mathrm{P} 2}=37.5 \mu \mathrm{m}$ in the $50 \mu \mathrm{m}$ long channel. ${ }^{9,70,71}$ The saturation transfer curves were measured in the condition $V_{\mathrm{ds}}=V_{\mathrm{gs}}-V_{\text {on }}$ (with $V_{\text {on }}$ being the onset voltage) to ensure a unipolar saturation regime throughout the entire gate voltage range. ${ }^{45}$ The onset voltages ( $V_{\text {on, h }}$ and $V_{\text {on, e }}$ ) were extracted from the linear transfer curves as the voltages for which the source-drain current rises above the background noise and gate-leakage current level for hole and electron transport respectively. The charge densities were obtained as $n=\frac{C_{\mathrm{i}}}{h} \cdot\left(V_{\mathrm{gs}}-V_{\mathrm{on}}\right)$, assuming the height of the accumulation layer to be $h=2 \mathrm{~nm} .^{41}$

\section{Associated Content}

\section{Supporting Information}

The Supporting Information is available free of charge on the ACS Publications website at DOI: Raman spectra of the deposited $(6,5)$ and $\mathrm{RN}$ network; atomic force microscopy images used to determine the SWCNT length distribution; photothermal deflection spectroscopy measurements of $(6,5)$ and RN networks; detailed outline of on-chip Seebeck coefficient measurements; onset-voltage ( $\left.V_{\text {on }}\right)$ extraction from linear transfer curves; capacitance measurements and device parameter overview; linear and saturation transfer curves of $(6,5)$-s-ttmgb network; comparison of 4pp linear and saturation mobilities; temperature and charge densitydependent Seebeck coefficients of all six networks; determination of the RN network composition based on the $\mathrm{E}_{22}$ peak of the absorption spectrum; parameterization of $\sigma^{\prime}(E)$ for $(6,5)$ s-ttmgb and RN-d-st networks; BTE simulations of the Seebeck coefficient considering onedimensional acoustic and optical phonon scattering; relationship of areal and linear SWCNT network density

\section{Author Information}

\section{Corresponding Authors}

Jana Zaumseil - Institute for Physical Chemistry, Universität Heidelberg, D-69120 Heidelberg, Germany;

Centre for Advanced Materials, Universität Heidelberg, D-69120 Heidelberg, Germany; http://orcid.org/0000-0002-2048-217X ;

E-mail: zaumseil@uni-heidelberg.de

Henning Sirringhaus - Cavendish Laboratory, University of Cambridge, Cambridge CB3 0HE, UK; https://orcid.org/0000-0001-98276061 ;

E-mail: hs220@cam.ac.uk

\section{Authors}

Martin Statz - Cavendish Laboratory, University of Cambridge, Cambridge CB3 0HE, UK; https://orcid.org/0000-0001-7791-3981

Severin Schneider - Institute for Physical Chemistry, Universität Heidelberg, D-69120 Heidelberg, Germany

Felix J. Berger - Institute for Physical Chemistry, Universität Heidelberg, D-69120 Heidelberg, Germany

Lianglun Lai - Cavendish Laboratory, University of Cambridge, Cambridge CB3 0HE, UK; Cambridge Graphene Centre, University of Cambridge, Cambridge CB3 0FA, UK

William A. Wood - Cavendish Laboratory, University of Cambridge, Cambridge CB3 0HE, UK

Mojtaba Abdi-Jalebi - Cavendish Laboratory, University of Cambridge, Cambridge CB3 0HE, UK;

Institute for Materials Discovery, University College London, London WC1E 7JE, UK; https://orcid.org/0000-0002-9430-6371

Simone Leingang - Institute for Inorganic Chemistry, Universität Heidelberg, D-69120 Heidelberg, Germany

Hans-Jörg Himmel - Institute for Inorganic Chemistry, Universität Heidelberg, D-69120 
Heidelberg, Germany; http://orcid.org/00000001-8111-3047

\section{Author Contributions}

M.S. developed and optimized the device structure and measurement setup. S.S. and F.J.B. fabricated all devices and performed all AFM and Raman measurements. M.S. performed all electric and thermoelectric measurements presented. L.L. and W.A.W. supported M.S. with the measurements. M.A.-J. performed the PDS measurements. S.L. and H.-J.H. synthesized the ttmgb molecules. M.S. performed all numerical simulations. M.S. developed and implemented the theoretical model with support from J.Z. and H.S.. H.S. directed and coordinated the research. J.Z. co-directed and coordinated the research. M.S., J.Z. and H.S. wrote the manuscript.

The authors declare no competing financial and non-financial interests.

\section{Acknowledgements}

We gratefully acknowledge financial support from the European Research Council (ERC) under the European Union's Horizon 2020 research and innovation programme (Grant agreement No. 817494 "TRIFECTs") and through an ERC Synergy Grant SC2 (No. 610115). M. Statz acknowledges the support from the Engineering and Physical Sciences Research Council (EPSRC) through an EPSRC studentship. S. Schneider, S. Leingang and H.-J. Himmel acknowledge funding by the Deutsche Forschungsgemeinschaft via the Collaborative Research Center "N-Heteropolycycles as Functional Materials" (SFB 1249, B03, C06). M. Abdi-Jalebi thanks the EPSRC for support as well as Cambridge Materials Limited and Wolfson College, University of Cambridge for their funding and technical support.

\section{References}

1. Franklin, A. D. Nanomaterials in Transistors: From High-Performance to Thin-Film Applications. Science 2015, 349, aab2750.

2. Zaumseil, J. Semiconducting Single-Walled Carbon Nanotubes or Very Rigid Conjugated Polymers: A Comparison. Adv. Electron. Mater. 2019, 5, 1800514.

3. Blackburn, J. L. Semiconducting SingleWalled Carbon Nanotubes in Solar Energy Harvesting. ACS Energy Lett. 2017, 2, 1598-1613.

4. Blackburn, J. L.; Ferguson, A. J.; Cho, C.; Grunlan, J. C. Carbon-Nanotube-Based Thermoelectric Materials and Devices. Adv. Mater. 2018, 30, 1704386.

5. Nish, A.; Hwang, J. Y.; Doig, J.; Nicholas, R. J. Highly Selective Dispersion of Single-Walled Carbon Nanotubes Using Aromatic Polymers. Nat. Nanotechnol. 2007, 2, 640-646.

6. Arnold, M. S.; Green, A. A.; Hulvat, J. F.; Stupp, S. I.; Hersam, M. C. Sorting Carbon Nanotubes by Electronic Structure Using Density Differentiation. Nat. Nanotechnol. 2006, 1, 60-65.

7. Yang, Y.; Ding, L.; Han, J.; Zhang, Z.; Peng, L.-M. High-Performance Complementary Transistors and Medium-Scale Integrated Circuits Based on Carbon Nanotube Thin Films. ACS Nano 2017, 11, 4124-4132.

8. Zhong, D.; Zhang, Z.; Ding, L.; Han, J.; Xiao, M.; Si, J.; Xu, L.; Qiu, C.; Peng, L.M. Gigahertz Integrated Circuits Based on Carbon Nanotube Films. Nat. Electron. 2018, 1, 40-45.

9. Brohmann, M.; Rother, M.; Schießl, S. P.; Preis, E.; Allard, S.; Scherf, U.; Zaumseil, J. Temperature-Dependent Charge Transport in Polymer-Sorted Semiconducting Carbon Nanotube Networks with Dif- 
ferent Diameter Distributions. J. Phys. Chem. C 2018, 122, 19886-19896.

10. Blackburn, J. L.; Kang, S. D.; Roos, M. J.; Norton-Baker, B.; Miller, E. M.; Ferguson, A. J. Intrinsic and Extrinsically Limited Thermoelectric Transport Within Semiconducting Single-Walled Carbon Nanotube Networks. Adv. Electron. Mater. 2019, 5, 1800910.

11. Sheng, P. Fluctuation-Induced Tunneling Conduction in Disordered Materials. Phys. Rev. B 1980, 21, 2180-2195.

12. Kaiser, A. B.; Park, Y. W.; Kim, G. T.; Choi, E. S.; Düsberg, G.; Roth, S. Electronic Transport in Carbon Nanotube Ropes and Mats. Synth. Met. 1999, 103, 2547-2550.

13. Kim, G.; Choi, E.; Kim, D.; Suh, D.; Park, Y.; Liu, K.; Duesberg, G.; Roth, S. Magnetoresistance of an Entangled SingleWall Carbon-Nanotube Network. Phys. Rev. B 1998, 58, 16064-16069.

14. Hu, L.; Hecht, D. S.; Grüner, G. Percolation in Transparent and Conducting Carbon Nanotube Networks. Nano Lett. 2004, 4, 2513-2517.

15. Hone, J.; Ellwood, I.; Muno, M.; Mizel, A.; Cohen, M. L.; Zettl, A.; Rinzler, A. G.; Smalley, R. E. Thermoelectric Power of Single-Walled Carbon Nanotubes. Phys. Rev. Lett. 1998, 80, 1042-1045.

16. Hung, N. T.; Nugraha, A. R.; Hasdeo, E. H.; Dresselhaus, M. S.; Saito, R. Diameter Dependence of Thermoelectric Power of Semiconducting Carbon Nanotubes. Phys. Rev. B 2015, 92, 165426.

17. Rother, M.; Schießl, S. P.; Zakharko, Y.; Gannott, F.; Zaumseil, J. Understanding Charge Transport in Mixed Networks of Semiconducting Carbon Nanotubes. ACS Appl. Mater. Interfaces 2016, 8, 55715579 .
18. Schießl, S. P.; De Vries, X.; Rother, M.; Massé, A.; Brohmann, M.; Bobbert, P. A.; Zaumseil; J. Modeling Carrier Density Dependent Charge Transport in Semiconducting Carbon Nanotube Networks. Phys. Rev. Mater. 2017, 1, 046003.

19. Shimotani, H.; Tsuda, S.; Yuan, H.; Yomogida, Y.; Moriya, R.; Takenobu, T.; Yanagi, K.; Iwasa, Y. Continuous BandFilling Control and One-Dimensional Transport in Metallic and Semiconducting Carbon Nanotube Tangled Films. $A d v$. Funct. Mater. 2014, 24, 3305-3311.

20. Shimizu, S.; Iizuka, T.; Kanahashi, K.; $\mathrm{Pu}$, J.; Yanagi, K.; Takenobu, T.; Iwasa, Y. Thermoelectric Detection of Multi-Subband Density of States in Semiconducting and Metallic Single-Walled Carbon Nanotubes. Small 2016, 12, 33883392 .

21. Zorn, N. F.; Scuratti, F.; Berger, F. J.; Perinot, A.; Heimfarth, D.; Caironi, M.; Zaumseil, J. Probing Mobile Charge Carriers in Semiconducting Carbon Nanotube Networks by Charge Modulation Spectroscopy. ACS Nano 2020, 14, 2412-2423.

22. Perebeinos, V.; Tersoff, J.; Avouris, P. Electron-Phonon Interaction and Transport in Semiconducting Carbon Nanotubes. Phys. Rev. Lett. 2005, 94, 086802.

23. Zhou, X.; Park, J. Y.; Huang, S.; Liu, J.; McEuen, P. L. Band Structure, Phonon Scattering, and the Performance Limit of Single-Walled Carbon Nanotube Transistors. Phys. Rev. Lett. 2005, 95, 146805.

24. Schneider, S.; Brohmann, M.; Lorenz, R.; Hofstetter, Y. J.; Rother, M.; Sauter, E.; Zharnikov, M.; Vaynzof, Y.; Himmel, H.J.; Zaumseil, J. Efficient $N$-Doping and Hole Blocking in Single-Walled Carbon Nanotube Transistors with 1,2,4,5Tetrakis(tetramethylguanidino)benzene. ACS Nano 2018, 12, 5895-5902. 
25. Fritzsche, H. A General Expression for the Thermoelectric Power. Solid State Commun. 1971, 9, 1813-1815.

26. Statz, M.; Venkateshvaran, D.; Jiao, X.; Schott, S.; McNeill, C. R.; Emin, D.; Sirringhaus, H.; Di Pietro, R. On the Manifestation of Electron-Electron Interactions in the Thermoelectric Response of Semicrystalline Conjugated Polymers with Low Energetic Disorder. Commun. Phys. 2018, 1, 16.

27. Kaiser, A. B. Thermoelectric Power and Conductivity of Heterogeneous Conducting Polymers. Phys. Rev. B 1989, 40, 28062813.

28. Arkhipov, V. I.; Heremans, P.; Emelianova, E. V.; Adriaenssens, G. J.; Bässler, H. Charge Carrier Mobility in Doped Semiconducting Polymers. Appl. Phys. Lett. 2003, 82, 3245-3247.

29. Saito, R.; Dresselhaus, G. Trigonal Warping Effect of Carbon Nanotubes. Phys. Rev. B 2000, 61, 2981-2990.

30. Maruyama, S. 1D DOS (Van Hove Singularity). http://www.photon.t.u-tokyo . ac.jp/ maruyama/kataura/1D_DOS.html (accessed 10/07/2020).

31. Guo, J. A Quantum-Mechanical Treatment of Phonon Scattering in Carbon Nanotube Transistors. J. Appl. Phys. 2005, 98, 063519 .

32. Popov, V. N.; Lambin, P. Intraband Electron-Phonon Scattering in SingleWalled Carbon Nanotubes. Phys. Rev. B 2006, 74, 075415.

33. Ozawa, H.; Ide, N.; Fujigaya, T.; Niidome, Y.; Nakashima, N. One-Pot Separation of Highly Enriched (6,5)Single-Walled Carbon Nanotubes Using a Fluorene-Based Copolymer. Chem. Lett. 2011, 40, 239-241.
34. Mistry, K. S.; Larsen, B. A.; Blackburn, J. L. High-Yield Dispersions of LargeDiameter Semiconducting Single-Walled Carbon Nanotubes with Tunable Narrow Chirality Distributions. ACS Nano 2013, 7, 2231-2239.

35. Graf, A.; Zakharko, Y.; Schießl, S. P.; Backes, C.; Pfohl, M.; Flavel, B. S.; Zaumseil, J. Large Scale, Selective Dispersion of Long Single-Walled Carbon Nanotubes with High Photoluminescence Quantum Yield by Shear Force Mixing. Carbon 2016, 105, 593-599.

36. Yu, H.; Hermann, S.; Schulz, S. E.; Gessner, T.; Dong, Z.; Li, W. J. Optimizing Sonication Parameters for Dispersion of SingleWalled Carbon Nanotubes. Chem. Phys. 2012, 408, 11-16.

37. Kim, W.; Javey, A.; Vermesh, O.; Wang, Q.; Li, Y.; Dai, H. Hysteresis Caused by Water Molecules in Carbon Nanotube Field-Effect Transistors. Nano Lett. 2003, 3, 193-198.

38. Aguirre, C. M.; Levesque, P. L.; Paillet, M.; Lapointe, F.; St-Antoine, B. C.; Desjardins, P.; Martel, R. The Role of the Oxygen/Water Redox Couple in Suppressing Electron Conduction in Field-Effect Transistors. Adv. Mater. 2009, 21, 30873091.

39. Schießl, S. P.; Rother, M.; Lüttgens, J.; Zaumseil, J. Extracting the Field-Effect Mobilities of Random Semiconducting Single-Walled Carbon Nanotube Networks: A Critical Comparison of Methods. Appl. Phys. Lett. 2017, 111, 193301.

40. Urbach, F. The Long-Wavelength Edge of Photographic Sensitivity and of the Electronic Absorption of Solids. Phys. Rev. 1953, 92, 1324.

41. Kronemeijer, A. J.; Pecunia, V.; Venkateshvaran, D.; Nikolka, M.; Sadhanala, A.; Moriarty, J.; Szumilo, M.; Sirringhaus, H. Two-Dimensional Carrier 
Distribution in Top-Gate Polymer FieldEffect Transistors: Correlation Between Width of Density of Localized States and Urbach Energy. Adv. Mater. 2014, 26, 728-733.

42. Venkateshvaran, D.; Nikolka, M.; Sadhanala, A.; Lemaur, V.; Zelazny, M.; Kepa, M.; Hurhangee, M.; Kronemeijer, A. J.; Pecunia, V.; Nasrallah, I.; Romanov, I.; Broch, K.; McCulloch, I.; Emin, D.; Olivier, Y.; Cornil, J.; Beljonne, D.; Sirringhaus, H. Approaching Disorder-Free Transport in High-Mobility Conjugated Polymers. Nature 2014, 515, 384-388.

43. Weisman, R. B.; Bachilo, S. M. Dependence of Optical Transition Energies on Structure for Single-Walled Carbon Nanotubes in Aqueous Suspension: An Empirical Kataura Plot. Nano Lett. 2003, 3, 1235-1238.

44. Held, M.; Schießl, S. P.; Miehler, D.; Gannott, F.; Zaumseil, J. Polymer/Metal Oxide Hybrid Dielectrics for Low Voltage FieldEffect Transistors with Solution-Processed, High-Mobility Semiconductors. Appl. Phys. Lett. 2015, 107, 083301.

45. Di Pietro, R.; Venkateshvaran, D.; Klug, A.; List-Kratochvil, E. J.; Facchetti, A.; Sirringhaus, H.; Neher, D. Simultaneous Extraction of Charge Density Dependent Mobility and Variable Contact Resistance from Thin Film Transistors. Appl. Phys. Lett. 2014, 104, 193501.

46. Heikes, R. R.; Ure, R. W. Thermoelectricity. Science and Engineering. (1st ed.); Interscience Publishers: New York, London, 1961; pp 75-82.

47. Brohmann, M.; Berger, F. J.; Matthiesen, M.; Schießl, S. P.; Schneider, S.; Zaumseil, J. Charge Transport in Mixed Semiconducting Carbon Nanotube Networks with Tailored Mixing Ratios. ACS Nano 2019, 13, 7323-7332.
48. Vissenberg, M. C.; Matters, M. Theory of the Field-Effect Mobility in Amorphous Organic Transistors. Phys. Rev. B 1998, 5\%, 12964-12967.

49. Di Pietro, R.; Nasrallah, I.; Carpenter, J.; Gann, E.; Kölln, L. S.; Thomsen, L.; Venkateshvaran, D.; O'Hara, K.; Sadhanala, A.; Chabinyc, M.; McNeill, C. R.; Facchetti, A.; Ade, H.; Sirringhaus, H.; Neher, D. Coulomb Enhanced Charge Transport in Semicrystalline Polymer Semiconductors. Adv. Funct. Mater. 2016, 26, 8011-8022.

50. Blackburn, J. L.; Barnes, T. M.; Beard, M. C.; Kim, Y. H.; Tenent, R. C.; McDonald, T. J.; To, B.; Coutts, T. J.; Heben, M. J. Transparent Conductive Single-Walled Carbon Nanotube Networks with Precisely Tunable Ratios of Semiconducting and Metallic Nanotubes. ACS Nano 2008, 2, 1266-1274.

51. Gao, J.; Loo, Y.-L. TemperatureDependent Electrical Transport in Polymer-Sorted Semiconducting Carbon Nanotube Networks. Adv. Funct. Mater. 2015, 25, 105-110.

52. Ferguson, A. J.; Reid, O. G.; Nanayakkara, S. U.; Ihly, R.; Blackburn, J. L. Efficiency of Charge-Transfer Doping in Organic Semiconductors Probed with Quantitative Microwave and DirectCurrent Conductance. J. Phys. Chem. Lett. 2018, 9, 6864-6870.

53. Znidarsic, A.; Kaskela, A.; Laiho, P.; Gaberscek, M.; Ohno, Y.; Nasibulin, A. G.; Kauppinen, E. I.; Hassanien, A. Spatially Resolved Transport Properties of Pristine and Doped Single-Walled Carbon Nanotube Networks. J. Phys. Chem. C 2013, 117, 13324-13330.

54. Kirkpatrick, S. Percolation and Conduction. Rev. Mod. Phys. 1973, 45, 574-588.

55. Bernasconi, J. Electrical Conductivity in Disordered Systems. Phys. Rev. B 1973, 7, 2252-2260. 
56. Yamamoto, T.; Fukuyama, H. Possible High Thermoelectric Power in Semiconducting Carbon Nanotubes - A Case Study of Doped One-Dimensional Semiconductors. J. Phys. Soc. Jpn. 2018, 87, 024707.

57. Ashcroft, N. W.; Mermin, N. D. Solid State Physics (33rd ed.); Holt, Rinehart and Winston: New York, 1976; pp 243-258.

58. Wong, H.-S. P.; Akinwande, D. Carbon Nanotube and Graphene Device Physics (1st ed.); Cambridge University Press: Cambridge, 2010; pp 105-117.

59. Kang, S. D.; Snyder, G. J. ChargeTransport Model for Conducting Polymers. Nat. Mater. 2017, 16, 252-257.

60. Tanaka, Y.; Hirana, Y.; Niidome, Y.; Kato, K.; Saito, S.; Nakashima, N. Experimentally Determined Redox Potentials of Individual $(\mathrm{n}, \mathrm{m})$ Single-Walled Carbon Nanotubes. Angew. Chem. Int. Ed. 2009, 48, 7655-7659.

61. Hwang, E. H.; Das Sarma, S. Acoustic Phonon Scattering Limited Carrier Mobility in Two-Dimensional Extrinsic Graphene. Phys. Rev. B 2008, 77, 115449.

62. Kaasbjerg, K.; Thygesen, K. S.; Jacobsen, K. W. Unraveling the Acoustic Electron-Phonon Interaction in Graphene. Phys. Rev. B 2012, 85, 165440.

63. Li, H.; Gordeev, G.; Garrity, O.; Peyyety, N. A.; Selvasundaram, P. B.; Dehm, S.; Krupke, R.; Cambré, S.; Wenseleers, W.; Reich, S.; Zheng, M.; Fagan, J. A.; Flavel, B. S. Separation of Specific Single-Enantiomer Single-Wall Carbon Nanotubes in the Large-Diameter Regime. ACS Nano 2020, 14, 948-963.

64. Snyder, G. J.; Toberer, E. S. Complex Thermoelectric Materials. Nat. Mater. 2008, 7, 105-114.

65. Macleod, B. A.; Stanton, N. J.; Gould, I. E.; Wesenberg, D.; Ihly, R.; Owczarczyk, Z. R.; Hurst, K. E.; Fewox, C. S.; Folmar, C. N.;
Holman Hughes, K.; Zink, B. L.; Blackburn, J. L.; Ferguson, A. J. Large $N$ - and $P$-Type Thermoelectric Power Factors from Doped Semiconducting Single-Walled Carbon Nanotube Thin Films. Energy Environ. Sci. 2017, 10, 2168-2179.

66. Avery, A. D.; Zhou, B. H.; Lee, J.; Lee, E.-S.; Miller, E. M.; Ihly, R.; Wesenberg, D.; Mistry, K. S.; Guillot, S. L.; Zink, B. L.; Kim, Y.-H.; Blackburn, J. L.; Ferguson, A. J. Tailored Semiconducting Carbon Nanotube Networks with Enhanced Thermoelectric Properties. Nat. Energy 2016, 1, 1-9.

67. Peters, A.; Kaifer, E.; Himmel, H.-J. 1,2,4,5Tetrakis(tetramethylguanidino)benzene: Synthesis and Properties of a New Molecular Electron Donor. Eur. J. Org. Chem. 2008, 2008, 5907-5914.

68. Himmel, H.-J. Guanidines as Reagents in Proton-Coupled Electron Transfer Reactions and Redox Catalysts. Synlett 2018, 29, 1957-1977.

69. Jackson, W. B.; Amer, N. M.; Boccara, A. C.; Fournier, D. Photothermal Deflection Spectroscopy and Detection. Appl. Opt. 1981, 20, 1333-1344.

70. Liu, C.; Li, G.; Di Pietro, R.; Huang, J.; Noh, Y. Y.; Liu, X.; Minari, T. Device Physics of Contact Issues for the Overestimation and Underestimation of Carrier Mobility in Field-Effect Transistors. Phys. Rev. Appl. 2017, 8, 034020.

71. Choi, H. H.; Cho, K.; Frisbie, C. D.; Sirringhaus, H.; Podzorov, V. Critical Assessment of Charge Mobility Extraction in FETs. Nat. Mater. 2018, 17, 2-7. 


\section{Graphical TOC Entry}

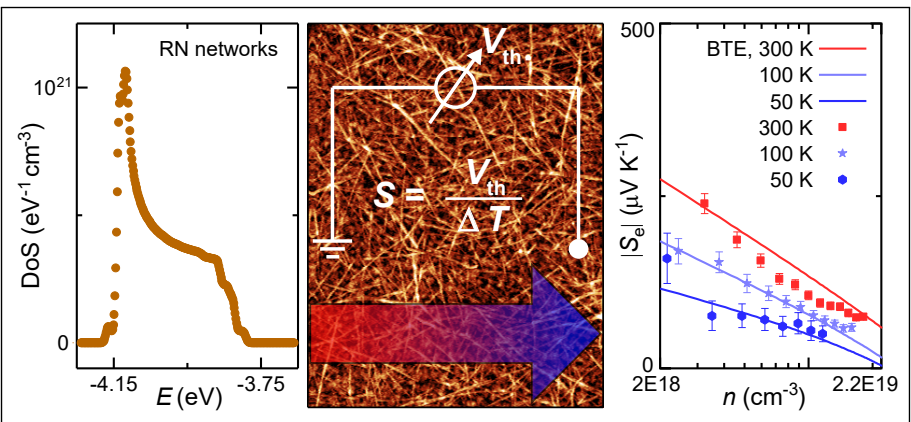

